\title{
A Taylor basis for kinematic nonlinear realtime simulations. Part II: The Taylor basis
}

\author{
Andersen, Sebastian; Poulsen, Peter Noe
}

Published in:

Earthquake Engineering and Structural Dynamics

Link to article, DOI:

10.1002/eqe.3175

Publication date:

2019

Document Version

Peer reviewed version

Link back to DTU Orbit

Citation (APA):

Andersen, S., \& Poulsen, P. N. (2019). A Taylor basis for kinematic nonlinear realtime simulations. Part II: The Taylor basis. Earthquake Engineering and Structural Dynamics, 48(8), 929-948.

https://doi.org/10.1002/eqe.3175

\section{General rights}

Copyright and moral rights for the publications made accessible in the public portal are retained by the authors and/or other copyright owners and it is a condition of accessing publications that users recognise and abide by the legal requirements associated with these rights.

- Users may download and print one copy of any publication from the public portal for the purpose of private study or research.

- You may not further distribute the material or use it for any profit-making activity or commercial gain

- You may freely distribute the URL identifying the publication in the public portal

If you believe that this document breaches copyright please contact us providing details, and we will remove access to the work immediately and investigate your claim. 


\title{
A Taylor Basis for Kinematic Nonlinear Real-time Simulations. Part II: The Taylor Basis
}

\author{
S. Andersen ${ }^{* \dagger}$ and P. N. Poulsen ${ }^{\ddagger}$ \\ Department of Civil Engineering, Technical University of Denmark, 2800 Kgs. Lyngby, Denmark
}

\begin{abstract}
SUMMARY
Real-time simulations are used to a significant extent in many engineering fields. However, if nonlinearities are included, the real-time requirement significantly limits the size and complexity of numerical models. The present work constitutes the second of two papers where a general method to simulate kinematic nonlinear structures more efficiently is introduced. In the present work, an efficient basis formulation that enables the number of basis vectors to be increased without increasing the number of unknown basis co-ordinates is presented. This allows for larger numerical kinematically nonlinear models to run in real time. The basis is organized from a Taylor series that includes the system mode shapes and their complete first order modal derivatives derived in Part I. The Taylor series predicts fixed linear relations between the modal co-ordinates of the system mode shapes and the modal derivatives, respectively. Thus, the full solution is known solely by determining the modal co-ordinates of the mode shapes, which significantly minimizes the computational costs. Furthermore, it is illustrated that the stability of the Taylor basis is dependent on the mode shape frequencies only, allowing the applied time steps to be significantly larger than in standard nonlinear basis analysis. An example illustrates a case where the computational time can be decreased by one order of magnitude using a Taylor basis formulation compared to a standard basis formulation including identical basis vectors.
\end{abstract}

Received ...

KEY WORDS: Taylor Basis; Modal Derivatives; Kinematic Nonlinearities; Basis Projection; Finite Element Analysis; Real-time Simulations

\section{INTRODUCTION}

The use of real-time simulations in the industry and research community within engineering fields is increasing. Real-time simulations are e.g. used in computer graphics, medical applications and structural testing, see [1-3].

The motivation for the present work relates to the field of hybrid testing, where structural full scale tests are divided in a physical substructure and an analytical substructure which are coupled through a continuously interchange of data. The concept of hybrid testing was developed by Japanese scientists in the 1960s as an alternative to shaking tables for seismic testing, see [4]. The structural behavior of the physical substructure is considered a black box and thus, must be tested physically. The behavior of the remaining part of the structure is known and is modeled numerically, typically by the finite element method (FEM). If the physical substructure is rate-dependent both the physical test and the numerical simulations are performed in real time, and the test is often referred to as

*Correspondence to: Department of Civil Engineering, Technical University of Denmark, 2800 Kgs. Lyngby, Denmark. E-mail: seba@byg.dtu.dk

$\dagger$ Postdoc

${ }^{\ddagger}$ Associate Professor 
real-time hybrid simulation (RTHS). For a more in-depth description of the principles behind hybrid testing, see e.g. [5-10].

The present work constitutes the second of two papers with the overall aim to formulate a general method for improving the simulations of kinematically nonlinear structures in real time. The focus is purely numerical, and coupling of numerical and physical substructures which appears in e.g. RTHS is not considered.

Despite of the computational power available today, the size and complexity of nonlinear numerical models capable of running in real-time are very restricted. The size of nonlinear numerical models included in a nonlinear RTHS e.g. is relatively modest with a maximum of 514 applied degrees of freedom (DOFs), see [11]. One of the critical reasons for the size limitations of real-time FEM simulations is the time consuming evaluation of the internal nodal restoring forces performed element-by-element followed by assembling of the global set of equations in each time step. Thus, this is a key area to consider if the computational efficiency should be improved.

A very efficient tool to improve the computational efficiency in numerical simulations is basis projection. This method is widely used and it also constitutes the focus of the present work. Its first application for kinematically nonlinear structures can be dated back to the work in [12]. Basis projection is also used in real-time application, e.g. in the field of computer graphics simulations [1], for medical purposes [2] and also for linear and nonlinear RTHS [13, 14].

Among some of the most used basis vectors for kinematic nonlinear systems are the linear normal modes (also known as the system mode shapes) and the modal derivatives (MDs), see [15]. The MDs were initially introduced in $[16,17]$ by differentiating the linearized eigenvalue problem. However, the differentiated equations only govern a set of approximate modal derivatives. Furthermore, the equations include a singular matrix, which requires the introduction of some assumptions before they can be solved. To resolve these singularity issues many modifications have been suggested, see e.g. [1,18-23]. However, common to all of the suggestions presented, is that they represent some kind of approximation. The approximation issue was, however, solved in part I of the present work where a set of equations governing the complete MDs were derived by use of perturbation methods.

The aim of the present work is to develop an efficient and accurate method for real-time simulations of kinematically nonlinear numerical systems. The method is based on basis projection including system mode shapes and MDs. In Part I the focus was to derive a novel set of equations governing the complete first order MDs by use of perturbation methods. In the present paper the complete MDs derived in Part I are then used in a so-called Taylor basis to significantly reduce the computational time when simulating kinematic nonlinear systems. The basis formulation predicts fixed linear relations between the modal co-ordinates of the system mode shapes and the MDs, respectively. This allows the latter to be included in the basis without introducing further unknown modal co-ordinates. Thus, the full solution is known solely by determining the modal co-ordinates of the system mode shapes. Furthermore, it is shown that the stability of the Taylor basis including the complete MDs only depends on the frequencies belonging to the system mode shapes, and not on the significantly higher frequencies related to the MDs, which is the case in standard time integration schemes. This allows the time steps to be increased significantly compared to standard time integration methods.

In [23] the principle of including MDs in a Taylor basis with fixed relations in the unknown modal co-ordinates was also applied. The approach showed to improve the computational efficiency. However, the MDs were evaluated as approximations from several nonlinear static displacement fields. The present work distinguishes itself from the work in [23] by including the complete modal derivatives in a general manner. Furthermore, the present work investigates the improvements with respect to computational time in more detail and it contributes with a stability analysis of the basis formulation.

To further improve the computational speed, a reformulation technique introduced by [24] is also incorporated to speed up the assembling of the nonlinear equations of motion in each integration time step. The concept consists of reformulating the global internal restoring forces as a sum of constant equivalent force vectors multiplied by the modal co-ordinates. The technique has been applied by several researchers to reduce the simulation times, see e.g. [1,25-27]. 
In the following section the principle of basis projection using both a so-called standard basis formulation and a Taylor basis formulation is presented. Next, in Section 3 it is described in general how the nonlinear restoring forces are formulated as a sum of constant equivalent force vectors by use of the reformulation technique suggested in [24]. Then, in Section 4 the significant computational efficiency and stability of the Taylor basis is illustrated by considering an example with a highly nonlinear frame. Finally, a conclusion is given in Section 5.

\section{BASIS PROJECTION OF THE KINEMATIC NONLINEAR EQUATIONS OF MOTION}

By use of Calculus of Variation, applied as the principle of virtual work, any set of governing equations of motion (EOMs) including kinematic nonlinearities can be organized in discrete form. A general formulation for the kinematic nonlinear EOMs with assumed constant mass and damping is

$$
\mathbf{M} \ddot{\mathbf{V}}+\mathbf{C} \dot{\mathbf{V}}+\mathbf{g}(\mathbf{V})=\mathbf{F}(t)
$$

where $\mathbf{V}$ is a $n \times 1$ global displacement vector containing $n$ DOFs. Differentiation with respect to time $t$ is defined through the notation $\dot{()}=d() / d t, \mathbf{M}$ and $\mathbf{C}$ are $n \times n$ mass and damping matrices, $\mathbf{F}(t)$ is the time-dependent external load vector of dimensions $n \times 1$ and $\mathbf{g}(\mathbf{V})$ is a $n \times 1$ vector representing the internal nodal restoring forces. Kinematic nonlinearities cause the vector $\mathbf{g}(\mathbf{V})$ to be a nonlinear function of the displacements.

In the following it is shown how the number of DOFs in the EOMs in (1) are reduced by use of basis projection, which can help to reduce the computational time spent on solving the equations. Two types of basis formulations are presented. The first one is referred to as a standard basis formulation, whereas the second is referred to as a Taylor basis formulation. The latter, including the complete MDs derived in Part I, constitutes the core of the present work.

\subsection{The Standard Basis Formulation}

In standard basis projection the number of DOFs are reduced by projecting the EOMs in (1) onto a basis organized as a linear relation between the physical and the reduced subspace co-ordinates. This is given as

$$
\mathbf{V}(\mathbf{s})=\mathbf{\Phi} \mathbf{s}
$$

where $\boldsymbol{\Phi}$ is a basis matrix of dimensions $n \times N$ with $N$ representing the number of basis vectors arranged as columns. Each basis vector has an associated unknown time dependent reduced subspace co-ordinate $s_{i}(t)=s_{i}, i \epsilon[1 ; N]$, and all of these co-ordinates are all collected in the vector s of dimensions $N \times 1$. In the following the basis formulation in (2) is referred to as a standard basis.

The discretized equations in (1) are projected onto the standard basis in (2) yielding the formulation

$$
\tilde{\mathbf{m}} \ddot{\mathbf{s}}+\tilde{\mathbf{c}} \dot{\mathbf{s}}+\tilde{\mathbf{g}}(\mathbf{s})=\tilde{\mathbf{f}}(t)
$$

with the projected modal matrices defined as

$$
\begin{aligned}
\tilde{\mathbf{m}} & =\boldsymbol{\Phi}^{T} \mathbf{M} \boldsymbol{\Phi} \\
\tilde{\mathbf{c}} & =\boldsymbol{\Phi}^{T} \mathbf{C} \boldsymbol{\Phi} \\
\tilde{\mathbf{g}}(\mathbf{s}) & =\boldsymbol{\Phi}^{T} \mathbf{g}(\boldsymbol{\Phi} \mathbf{s}) \\
\tilde{\mathbf{f}}(t) & =\boldsymbol{\Phi}^{T} \mathbf{F}(t)
\end{aligned}
$$

Typically, a relatively small set of basis vectors are required to obtain a good approximate solution for $\mathbf{V}$, by solving the projected equations in (3) for the unknown $\mathbf{s}$ and then transform this into physical co-ordinates by use of the relation in (2). The number of DOFs in (3) are typically significantly less compared to the full system in (1), that is, $N \ll n$. 
A natural choice for the basis vectors applied in (2) are the eigenvectors $\varphi_{i}$ fulfilling the linearized eigenvalue problem in (8) with the tangent stiffness matrix, $\mathbf{K}_{T}(\mathbf{V})$, the mass matrix, $\mathbf{M}$, and the $i$-th natural frequency, $\omega_{i}$ :

$$
\left(\mathbf{K}_{T}(\mathbf{V})-\omega_{i}^{2} \mathbf{M}\right) \boldsymbol{\varphi}_{i}=\mathbf{0}
$$

The eigenvectors, $\varphi_{i}$, are also known as the system mode shapes. In the following these are simply referred to as the mode shapes.

The mode shapes that contribute most significantly to the response of linear systems are typically those with frequencies in the domain of the excitation frequencies and with relatively high absolute modal load contributions, which are given as $\left|\tilde{f}_{i}(t)\right|=\left|\boldsymbol{\varphi}_{i}^{T} \mathbf{F}(t)\right|$, where $|()|$ indicates the absolute value. These are often represented by the mode shapes with the lowest frequencies. The same mode shapes are also significant to the response of kinematically nonlinear systems. However, in nonlinear structures, basis vectors that represent coupling activated displacements are also of high importance. These are often characterized as modes with small modal loads and frequencies significantly higher than the excitation frequencies, see e.g. Part I. If these are not included, the system may experience so-called membrane locking, which leads to poorly predicted displacements and/or unstable systems, see [28]. In Part I it was shown that the first order coupling-effects for a system with zero initial displacements are represented by the modal derivatives $\partial \varphi_{i} / \partial s_{j}$ governed by the system of equations

$$
\left[\mathbf{K}_{T}(\mathbf{0})-\left(\omega_{j 0}+\omega_{i 0}\right)^{2} \mathbf{M}\right] \frac{1}{2}\left(\frac{\partial \boldsymbol{\varphi}_{i 0}}{\partial s_{j}}+\frac{\partial \boldsymbol{\varphi}_{j 0}}{\partial s_{i}}\right)=\left[\alpha_{0} \mathbf{M}-\frac{\partial \mathbf{K}_{S}(\mathbf{0})}{\partial s_{i}}\right] \boldsymbol{\varphi}_{j 0}
$$

with the subindex 0 referring to the initial state with zero displacements. On the left-hand side of (9) the sum of two first order modal derivatives with switched indices appear. These figures are multiplied by the tangent stiffness matrix, $\mathbf{K}_{T}(\mathbf{V})$, at the zero deformation state $(\mathbf{V}=\mathbf{0})$, subtracted by the product of the mass matrix times the squared sum of the $j$-th and $i$-th natural frequencies. The right hand side consist of a matrix containing the mass matrix multiplied by a coefficient denoted $\alpha_{0}$ minus the secant stiffness, $\mathbf{K}_{S}(\mathbf{V})$, differentiated with respect to the $i$-th modal co-ordinate and evaluated at zero displacements. The matrix is multiplied onto the $j$-th mode shape. The coefficient $\alpha_{0}$ is given in (10), where it is seen to be a function of the mass matrix, the $j$-th and $i$-th mode shapes and the secant stiffness matrix differentiated with respect to the $j$-th and $i$-th modal co-ordinates, respectively.

$$
\alpha_{0}=\frac{\boldsymbol{\varphi}_{j 0}^{T}}{\boldsymbol{\varphi}_{j 0}^{T} \mathbf{M} \boldsymbol{\varphi}_{j 0}}\left(\frac{\partial \mathbf{K}_{S}(\mathbf{0})}{\partial s_{i}} \boldsymbol{\varphi}_{j 0}-\frac{\partial \mathbf{K}_{S}(\mathbf{0})}{\partial s_{j}} \boldsymbol{\varphi}_{i 0}\right)
$$

It is important to stress that the modal derivatives in (9) can only be determined as a sum of two vectors. A compact formulation for this is introduced as

$$
\partial \varphi_{i j}=\frac{\partial \varphi_{i}}{\partial s_{j}}+\left(1-\delta_{i j}\right) \frac{\partial \varphi_{j}}{\partial s_{i}} \quad, \quad i, j \epsilon[1 ; N] \wedge j \leq i
$$

Given $N$ mode shapes then $H=\frac{1}{2}\left(N+N^{2}\right)$ is the number of combinations of the modal derivatives $\partial \varphi_{i j}$. Including all of the $N$ mode shapes and the corresponding $H$ modal derivatives in the standard basis formulation in (2), this may be written in the form 


$$
\mathbf{V}(\mathbf{s})=\underbrace{\left[\begin{array}{lllllllll}
\boldsymbol{\varphi}_{1} & \boldsymbol{\varphi}_{2} & \ldots & \boldsymbol{\varphi}_{N} & \partial \boldsymbol{\varphi}_{11} & \partial \varphi_{21} & \partial \boldsymbol{\varphi}_{22} & \ldots & \partial \varphi_{N N}
\end{array}\right]}_{\mathbf{\Phi}}\left\{\begin{array}{c}
s_{1} \\
s_{2} \\
\vdots \\
s_{N} \\
s_{N+1} \\
s_{N+2} \\
s_{N+3} \\
\vdots \\
s_{N+H}
\end{array}\right\}
$$

with $N+H$ independent modal co-ordinates $s_{j}, j \epsilon[1 ; N+H]$. Examples of the use of a standard basis including modal derivatives are given in $[1,16]$ and in Part I. In the present work the standard basis is used as a reference when evaluating the precision, computational efficiency and stability of the so-called Taylor basis.

\subsection{The Taylor Basis Formulation}

In Part I the displacement field $\mathbf{V}(\mathbf{s})$ was expressed as a Taylor series in the modal co-ordinates s with $N$ mode shapes, $\varphi_{i}$, and $H$ modal derivatives, $\partial \varphi_{i j}$. For zero initial displacements the series reduced to

$$
\mathbf{V}(\mathbf{s})=\sum_{i=1}^{N} \boldsymbol{\varphi}_{i} s_{i}+\sum_{i=1}^{N} \sum_{j=1}^{i} \partial \boldsymbol{\varphi}_{i j} s_{i} s_{j}+\ldots
$$

The Taylor series in (13) suggests that the contribution from each of the mode shapes to the solution of $\mathbf{V}$ is given as the vector multiplied by its associated modal co-ordinate. Each of the first order MDs are multiplied by a second order product of the modal co-ordinates, consisting of the same coordinates as multiplied onto the mode shapes. Thus, including the MDs does not introduce further unknowns into the system. The Taylor series, therefore, presents a more efficient way to include the MDs. The mode shapes and the first order MDs in (13) are organized in the matrix format

$$
\boldsymbol{\Phi}_{T}=\left[\boldsymbol{\varphi}_{1}, \quad \boldsymbol{\varphi}_{2}, \quad \ldots \quad \boldsymbol{\varphi}_{N}, \quad \partial \boldsymbol{\varphi}_{11}, \quad \partial \boldsymbol{\varphi}_{21}, \quad \partial \boldsymbol{\varphi}_{22}, \quad \partial \boldsymbol{\varphi}_{31}, \quad \partial \boldsymbol{\varphi}_{32}, \quad \ldots \quad \partial \boldsymbol{\varphi}_{N N}\right]
$$

which is of dimensions $n \times(N+H)$. In the following $\mathbf{\Phi}_{T}$ is referred to as the Taylor basis matrix. Moreover, the corresponding modal co-ordinates $s_{i}$ and the second order co-ordinate products $s_{i} s_{j}$ in (13) are collected in the vector

$$
\mathbf{s}_{T}=\left\{\begin{array}{c}
s_{1} \\
s_{2} \\
\vdots \\
s_{N} \\
s_{1} s_{1} \\
s_{2} s_{1} \\
s_{2} s_{2} \\
s_{3} s_{1} \\
s_{3} s_{2} \\
\vdots \\
s_{N} s_{N}
\end{array}\right\}
$$

which is of dimensions $(N+H) \times 1$. In the following the vector $\mathbf{s}_{T}$ is referred to as the Taylor co-ordinate vector. Given the definitions in (14) and (15) the Taylor series in (13), including the 
terms up to second order, can be given as the compact basis formulation

$$
\mathbf{V}(\mathbf{s})=\mathbf{\Phi}_{T} \mathbf{s}_{T}
$$

The formulation in (16) is referred to as the Taylor basis in the following. The Taylor basis matrix $\boldsymbol{\Phi}_{T}$ in (14) is identical to the basis matrix $\boldsymbol{\Phi}$ introduced in (12). However, the subscript $T$ is added to stress that it is part of the Taylor basis formulation including second order co-ordinate products in the Taylor co-ordinate vector $\mathbf{s}_{T}$. Projecting the EOMs in (1) onto the Taylor basis in (16) yields the formulation

$$
\hat{\mathbf{m}} \ddot{\mathbf{s}}_{T}+\hat{\mathbf{c}} \dot{\mathbf{s}}_{T}+\hat{\mathbf{g}}\left(\mathbf{s}_{T}\right)=\hat{\mathbf{f}}(t)
$$

with the following matrix definitions

$$
\begin{aligned}
\hat{\mathbf{m}} & =\left(\boldsymbol{\Phi}_{T}\right)^{T} \mathbf{M} \boldsymbol{\Phi}_{T} \\
\hat{\mathbf{c}} & =\left(\boldsymbol{\Phi}_{T}\right)^{T} \mathbf{C} \boldsymbol{\Phi}_{T} \\
\hat{\mathbf{g}}\left(\mathbf{s}_{T}\right) & =\left(\boldsymbol{\Phi}_{T}\right)^{T} \mathbf{g}\left(\boldsymbol{\Phi}_{T} \mathbf{s}_{T}\right) \\
\hat{\mathbf{f}}(t) & =\left(\boldsymbol{\Phi}_{T}\right)^{T} \mathbf{F}(t)
\end{aligned}
$$

and the following definitions of the velocity and acceleration vectors

$$
\dot{\mathbf{s}}_{T}=\left\{\begin{array}{c}
\dot{s}_{1} \\
\dot{s}_{2} \\
\vdots \\
\dot{s}_{N} \\
\dot{s}_{1} s_{1}+s_{1} \dot{s}_{1} \\
\dot{s}_{2} s_{1}+s_{2} \dot{s}_{1} \\
\dot{s}_{2} s_{2}+s_{2} \dot{s}_{2} \\
\dot{s}_{3} s_{1}+s_{3} \dot{s}_{1} \\
\vdots \\
\dot{s}_{N} s_{N}+s_{N} \dot{s}_{N}
\end{array}\right\}, \quad \ddot{\mathbf{s}}_{T}=\left\{\begin{array}{c}
\ddot{s}_{1} \\
\ddot{s}_{2} \\
\vdots \\
\ddot{s}_{N} \\
\ddot{s}_{1} s_{1}+2 \dot{s}_{1} \dot{s}_{1}+s_{1} \ddot{s}_{1} \\
\ddot{s}_{2} s_{1}+2 \dot{s}_{2} \dot{s}_{1}+s_{2} \ddot{s}_{1} \\
\ddot{s}_{2} s_{2}+2 \dot{s}_{2} \dot{s}_{2}+s_{2} \ddot{s}_{2} \\
\ddot{s}_{3} s_{1}+2 \dot{s}_{3} \dot{s}_{1}+s_{3} \ddot{s}_{1} \\
\vdots \\
\ddot{s}_{N} s_{N}+2 \dot{s}_{N} \dot{s}_{N}+s_{N} \ddot{s}_{N}
\end{array}\right\}
$$

From the Taylor co-ordinate vector in (15) and the accelerations and velocities in (22) all of the unknowns $\left(s_{i}, \dot{s}_{i}, \ddot{s}_{i}\right)$ are seen to appear multiple times. Therefore, the projected system of equations in (17) must be transformed from the $\left(\mathbf{s}_{T}, \dot{\mathbf{s}}_{T}, \ddot{\mathbf{s}}_{T}\right)$ co-ordinate system to the (s, $\left.\dot{\mathbf{s}}, \ddot{\mathbf{s}}\right)$ co-ordinate system before it is solved. This transformation is performed using straightforward mathematical approaches presented in the following section.

The Taylor basis formulation in (16) was also used in [23]. However, the MDs were taken as approximations determined from static displacement fields. In the present work the complete modal derivatives are determined directly from the complete governing system of equations in (9). Furthermore, it is shown that by including the complete modal derivatives in the Taylor basis, a significant increase in stability is achieved. Also a detailed investigation of the improvement in the simulation time is given here.

\subsection{Taylor Co-ordinate Transformation}

The projected system of equations in (17) is transformed from the $\left(\mathbf{s}_{T}, \dot{\mathbf{s}}_{T}, \ddot{\mathbf{s}}_{T}\right)$ co-ordinate system into the $(\mathbf{s}, \dot{\mathrm{s}}, \ddot{\mathbf{s}})$ co-ordinate system. For this, the projected EOMs are written in a variational form by multiplying it with the transposed virtual component vector $\delta \mathbf{s}_{T}$ :

$$
\left(\delta \mathbf{s}_{T}\right)^{T}\left(\hat{\mathbf{m}} \ddot{\mathbf{s}}_{T}+\hat{\mathbf{c}} \dot{\mathbf{s}}_{T}+\hat{\mathbf{g}}\left(\mathbf{s}_{T}\right)-\hat{\mathbf{f}}(t)\right)=0
$$


The vector $\delta \mathbf{s}_{T}$ is given as the variation of the vector $\mathbf{s}_{T}$ introduced in (15). This yields

$$
\delta \mathbf{s}_{T}=\left\{\begin{array}{c}
\delta s_{1} \\
\delta s_{2} \\
\vdots \\
\delta s_{N} \\
\delta s_{1} s_{1}+s_{1} \delta s_{1} \\
\delta s_{2} s_{1}+s_{2} \delta s_{1} \\
\delta s_{2} s_{2}+s_{2} \delta s_{2} \\
\vdots \\
\delta s_{N} s_{N}+s_{N} \delta s_{N}
\end{array}\right\}
$$

Next, a set of relations between the vector co-ordinates (s, $\dot{\mathbf{s}}, \ddot{\mathbf{s}}, \delta \mathbf{s})$ and $\left(\mathbf{s}_{T}, \dot{\mathbf{s}}_{T}, \ddot{\mathbf{s}}_{T}, \delta \mathbf{s}_{T}\right)$ are introduced:

$$
\begin{aligned}
\mathbf{s}_{T} & =\mathbf{Q}(\mathbf{s}) \mathbf{s} \\
\delta \mathbf{s}_{T} & =\mathbf{U}(\mathbf{s}) \delta \mathbf{s} \\
\dot{\mathbf{s}}_{T} & =\mathbf{U}(\mathbf{s}) \dot{\mathbf{s}} \\
\ddot{\mathbf{s}}_{T} & =\mathbf{U}(\mathbf{s}) \ddot{\mathbf{s}}+2 \mathbf{P}(\dot{\mathbf{s}}) \dot{\mathbf{s}}
\end{aligned}
$$

where $\mathbf{Q}(\mathbf{s}), \mathbf{U}(\mathbf{s})$ and $\mathbf{P}(\dot{\mathbf{s}})$ are transformation matrices of dimensions $(N+H) \times N$. Details on their formulation are presented in Appendix A. As indicated, the transformation matrices are functions of the modal displacement vector $\mathbf{s}$ and the modal velocity vector $\dot{\mathbf{s}}$. Inserting the relations in (25)-(28) into (23), the projected EOMs may be written in the (s, $\mathbf{s}, \ddot{\mathbf{s}})$ co-ordinates as:

$$
\overline{\mathbf{m}}(\mathbf{s}) \ddot{\mathbf{s}}+\overline{\mathbf{c}}(\mathbf{s}, \dot{\mathbf{s}}) \dot{\mathbf{s}}+\overline{\mathbf{g}}(\mathbf{s})=\overline{\mathbf{f}}(\mathbf{s}, t)
$$

with the following matrix and vector definitions

$$
\begin{aligned}
\overline{\mathbf{m}}(\mathbf{s}) & =\mathbf{U}(\mathbf{s})^{T} \hat{\mathbf{m}} \mathbf{U}(\mathbf{s}) \\
\overline{\mathbf{c}}(\mathbf{s}, \dot{\mathbf{s}}) & =\mathbf{U}(\mathbf{s})^{T}(2 \hat{\mathbf{m}} \mathbf{P}(\dot{\mathbf{s}})+\hat{\mathbf{c}} \mathbf{U}(\mathbf{s})) \\
\overline{\mathbf{g}}(\mathbf{s}) & =\mathbf{U}(\mathbf{s})^{T} \hat{\mathbf{g}}\left(\mathbf{s}_{T}\right) \\
\overline{\mathbf{f}}(\mathbf{s}, t) & =\mathbf{U}(\mathbf{s})^{T} \hat{\mathbf{f}}(t)
\end{aligned}
$$

After the transformation from the $\left(\mathbf{s}_{T}, \dot{\mathbf{s}}_{T}, \ddot{\mathbf{s}}_{T}\right)$ co-ordinate system into the (s, $\left.\dot{\mathbf{s}}, \ddot{\mathbf{s}}\right)$ co-ordinate system, the mass matrix, the damping matrix and the load vector in (30), (31) and (33) are now functions of the modal co-ordinates $\mathbf{s}$ and $\dot{\mathbf{s}}$. Furthermore, the damping matrix is seen to also be a function of the mass $\hat{\mathbf{m}}$.

\subsection{Linear Dependent Basis Vectors}

A possible scenario is that some of the included MDs will be linearly dependent either on some of the systems mode shapes or on some of the other included MDs, which was the case in the example in Part I. In such cases the rank of the basis matrices $\boldsymbol{\Phi}$ and $\boldsymbol{\Phi}_{T}$ in (12) and (16) is less than the number of basis vectors. To avoid singularity issues when solving for the unknowns, the linearly dependent vectors are removed from the standard basis matrix $\boldsymbol{\Phi}$ in (12), and their contribution is automatically accounted for by the remaining modes. However, when the Taylor basis in (16) is used, it is unnecessary to remove the linear dependent vectors from $\boldsymbol{\Phi}_{T}$, as the singularity issue is automatically resolved when the projected EOMs in (17) are transformed from the ( $\left.\mathbf{s}_{T}, \dot{\mathbf{s}}_{T}, \ddot{\mathbf{s}}_{T}\right)$ co-ordinate system into the (s, $\dot{\mathbf{s}}, \ddot{\mathbf{s}})$ co-ordinate system in (29).

In the process of identifying potential linear dependent basis vectors, a single modal derivative $\partial \varphi_{i j}$ is removed from the basis matrix $\Phi$ one at a time. The basis matrix without the modal derivative $\partial \varphi_{i j}$ is referred to as $\tilde{\boldsymbol{\Phi}}_{i j}$. The modal derivative which has been removed is then approximated by 
the linear expression

$$
\partial \boldsymbol{\varphi}_{i j} \approx \tilde{\boldsymbol{\Phi}}_{i j} \boldsymbol{\beta}_{i j}
$$

where $\boldsymbol{\beta}_{i j}$ is a vector containing a set of coefficients to be determined applying linear regression. As a measure for the error of the linear approximation in (34) a parameter $e$ is defined as

$$
e=\frac{\left\|\partial \boldsymbol{\varphi}_{i j}-\tilde{\boldsymbol{\Phi}}_{i j} \boldsymbol{\beta}_{i j}\right\|}{\left\|\partial \boldsymbol{\varphi}_{i j}\right\|}
$$

where $\|()\|$ indicates the length of a vector. If $e=0$, a set of coefficients $\boldsymbol{\beta}_{i j}$ exist such that the approximation in (34) is exact. In this case the modal derivative $\partial \boldsymbol{\varphi}_{i j}$ is said to be linear dependent on the basis vectors in $\tilde{\boldsymbol{\Phi}}_{i j}$. If $e>0$ the relation in (34) remains an approximation and the modal derivative is linearly independent on the basis vectors in $\tilde{\boldsymbol{\Phi}}_{i j}$. However, in order to account for numerical inaccuracies, the parameter $e$ should exceed $1 \%$ before the mode is said to be linearly independent. Thus, in the case that $\mathrm{e} \leq 1 \%$ the modal derivative under consideration is removed from the standard basis matrix $\Phi$ in (12) reducing the number of DOFs by one.

\section{POLYNOMIAL INTERNAL RESTORING FORCES}

The global internal restoring forces are formulated as a global vector polynomial in order to reduce the assembling time in each integration step. The principle was introduced in [24] and examples on the significant computational costs that can be saved by this polynomial formulation compared with standard element-by-element evaluations are given in [1,25-27].

In the following the global vector polynomial formulation of the internal restoring forces based on, respectively, the standard basis and the Taylor basis are presented. The two formulations are included in the simulations presented in Section 4 analyzing a highly nonlinear frame structure.

\subsection{Polynomial Restoring Forces}

The internal restoring forces of a kinematic nonlinear system can be expressed as a cubic expression in the displacement field V, see e.g. Part I:

$$
\mathbf{g}(\mathbf{V})=\left(\mathbf{K}_{0}+\mathbf{K}_{1}(\mathbf{V})+\mathbf{K}_{2}(\mathbf{V})\right) \mathbf{V}
$$

where $\mathbf{K}_{0}, \mathbf{K}_{1}(\mathbf{V})$ and $\mathbf{K}_{2}(\mathbf{V})$ are all stiffness matrices, which are either independent, linearly dependent or quadratically dependent on the displacement vector $\mathbf{V}$.

With the definition in (36) the projected internal restoring forces in (6) projected onto a standard basis formulation can, through simple mathematical derivations, be expressed as a cubic vector polynomial in the modal co-ordinates as

$$
\begin{aligned}
\tilde{\mathbf{g}}(\mathbf{s}) & =\boldsymbol{\Phi}^{T}\left(\mathbf{K}_{0}+\mathbf{K}_{1}(\mathbf{\Phi} \mathbf{s})+\mathbf{K}_{2}(\boldsymbol{\Phi} \mathbf{s})\right) \mathbf{\Phi} \mathbf{s} \\
& =\sum_{i=1}^{N} \mathbf{q}_{1, i} s_{i}+\sum_{i=1}^{N} \sum_{j=1}^{i} \mathbf{q}_{2, i j} s_{i} s_{j}+\sum_{i=1}^{N} \sum_{j=1}^{i} \sum_{k=1}^{j} \mathbf{q}_{3, i j k} s_{i} s_{j} s_{k}
\end{aligned}
$$

where $\mathbf{q}_{1, i}, \mathbf{q}_{2, i j}$ and $\mathbf{q}_{3, i j k}$ are referred to as the constant equivalent force vector coefficients. The definitions of these are presented in general form in Appendix B. The formulation in (37) is very convenient as it allows for all of the equivalent force vector coefficients to be arranged before starting the time integration. Thus, the usual time-consuming evaluation of the internal restoring force contributions performed element-by-element for every time step is replaced by a simple summation where only the modal co-ordinates vary.

Likewise, the internal restoring forces in (20) (projected onto a Taylor basis) may be formulated as a vector polynomial in the modal co-ordinates. However, as the Taylor co-ordinate vector $\mathbf{s}_{T}$ in (15) contains element co-ordinates of second order, the result is a sixth order polynomial in the 
reduced co-ordinates:

$$
\begin{aligned}
\hat{\mathbf{g}}\left(\mathbf{s}_{T}\right)= & \boldsymbol{\Phi}_{T}^{T}\left(\mathbf{K}_{0}+\mathbf{K}_{1}\left(\boldsymbol{\Phi}_{T} \mathbf{s}_{T}\right)+\mathbf{K}_{2}\left(\boldsymbol{\Phi}_{T} \mathbf{s}_{T}\right)\right) \mathbf{\Phi}_{T} \mathbf{s}_{T} \\
= & \sum_{i=1}^{N} \overline{\mathbf{q}}_{1, i} s_{i}+\sum_{i=1}^{N} \sum_{j=1}^{i} \overline{\mathbf{q}}_{2, i j} s_{i} s_{j}+\cdots+ \\
& \sum_{i=1}^{N} \sum_{j=1}^{i} \sum_{k=1}^{j} \sum_{l=1}^{k} \sum_{m=1}^{l} \sum_{n=1}^{m} \overline{\mathbf{q}}_{6, i j k l m n} s_{i} s_{j} s_{k} s_{l} s_{m} s_{n}
\end{aligned}
$$

where $\overline{\mathbf{q}}_{1, i}, \overline{\mathbf{q}}_{2, i j}, \ldots \overline{\mathbf{q}}_{6, i j k l m n}$ are constant equivalent force vector coefficients. A general approach used to identify the coefficients is presented in Appendix C.

\section{EXAMPLE - HIGHLY NONLINEAR FRAME}

In this section the computational efficiency, stability, and precision of the Taylor basis and the standard basis are considered. For this purpose an example with a kinematically highly nonlinear frame modeled using two-dimensional Euler-Bernoulli beam elements is studied. The simulations are executed with an in-house MATLAB code on a standard PC with an Intel Core i7-6500U processor with two cores, $2.5 \mathrm{GHz}$ and $16 \mathrm{~GB}$ RAM. It is important to stress, that the code cannot be considered optimized, and faster devices will (of course) improve the computational efficiency further. Despite of this, the results still serve as good indicators for the efficiency of the Taylor basis.

\subsection{Frame Geometry and Loading}

In Figure 1 a frame consisting of two equally long elements of length $\ell$ connected orthogonally to each other is shown. Both elements are characterized by a Young's modulus $E$, a density $\rho$, a solid circular cross-section with diameter $D$, a cross sectional area $A$ and a second moment of area $I$. For the sake of simplicity, the damping is set to zero. Moreover, the frame is simply supported. To amplify the kinematic nonlinear effects, the frame corner is restricted against horizontal displacements.

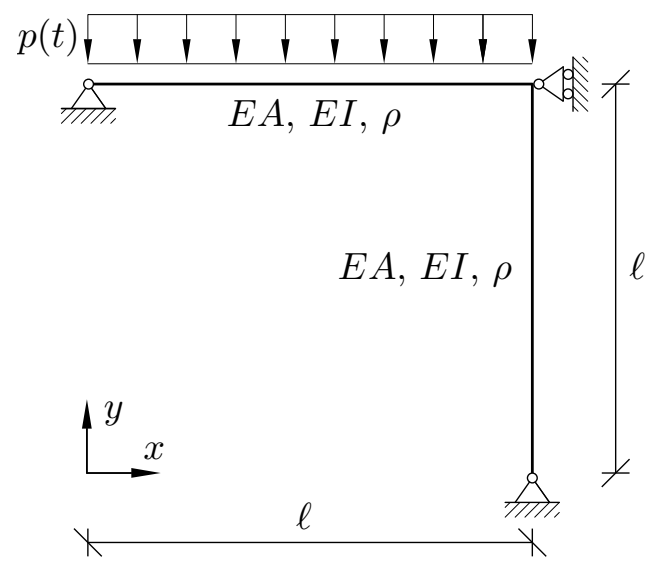

Figure 1. Nonlinear frame.

The horizontal frame element is loaded by a downwards, vertically oriented, harmonic line load, $p(t)$, with amplitude $F$ and frequency $\omega$ :

$$
p(t)=F \cdot \sin (\omega t)
$$

In Table I the applied load and frame parameters are presented. 
Table I. Frame parameters.

\begin{tabular}{cccc}
\hline Parameter & Unit & Formula & Magnitude \\
\hline$\ell$ & $\mathrm{m}$ & - & 10 \\
$E$ & $\mathrm{GPa}$ & - & 210 \\
$D$ & $\mathrm{~m}$ & - & 0.05 \\
$A$ & $\mathrm{~m}^{2}$ & $\frac{\pi}{4} D^{2}$ & $2 \cdot 10^{-3}$ \\
$I$ & $\mathrm{~m}^{4}$ & $\frac{\pi}{64} D^{4}$ & $3.07 \cdot 10^{-7}$ \\
$F$ & $\mathrm{~N} / \mathrm{m}$ & - & -3 \\
$\omega$ & $\mathrm{rad} / \mathrm{s}$ & & 6.40 \\
$\rho$ & $\mathrm{kg} / \mathrm{m}^{3}$ & - & 7800 \\
$c$ & $\mathrm{Ns} / \mathrm{m}$ & - & 0 \\
\hline
\end{tabular}

The horizontal and vertical displacements of the frame are denoted $u_{x}(x, y, t)$ and $u_{y}(x, y, t)$, respectively. In the following analyses the vertical displacement of the centre of the horizontal beam $u_{y}^{c}(t)=u_{y}(\ell / 2, \ell, t)$ and of the quarter point $u_{y}^{q}(t)=u_{y}(\ell / 4, \ell, t)$ are considered.

\subsection{Numerical Time Integration}

In the study of the efficiency, stability and precision of the standard basis and the Taylor basis formulations the central difference method (CDM) and the modified Euler method (MEM) are used. Both integration schemes are explicit, and they are chosen because explicit schemes by nature are better suited for performing real-time simulations compared to implicit methods. In order to check the results of the CDM and the MEM, the implicit Newmark integration method with parameters corresponding to the average acceleration case is also used. All three time integration schemes are implemented in a linear and a nonlinear version into the local MATLAB-based FEM program which was verified in Part I. Moreover, the frame is modelled by using plane Euler-Bernoulli beam elements introduced in Part I, with 20 elements in both the horizontal and vertical frame parts.

The damping matrix $\overline{\mathbf{c}}(\mathbf{s}, \dot{\mathbf{s}})$ included in the EOMs projected onto the Taylor basis in (29) is a function of both the modal displacement and velocity. However, in the CDM the velocity is not an explicit part of the formulation. Thus, in order to use the CDM an approximation for the discrete velocity is arranged from a Taylor series. The applied approximation is given as

$$
\dot{\mathbf{s}}^{(q)}=\frac{1}{\Delta t}\left(\frac{3}{2} \mathbf{s}^{(q)}-2 \mathbf{s}^{(q-1)}+\frac{1}{2} \mathbf{s}^{(q-2)}\right)
$$

where the upper indices $(q-n), n \epsilon[0 ; 2]$ refer to the discrete times $t^{(q-n)}=(q-n) \Delta t$ with $\Delta t$ representing the numerical time step magnitude. Furthermore, $q$ is a positive integer. Full details on the derivations of the expression in (40) are given in Appendix D.

In Algorithm 1 the CDM based integration scheme used for the Taylor basis simulations is presented. It is divided into thirteen steps, each explained by a short text accompanied by references to the relevant formulas and/or appendices. Step one to five cover the pre-processing tasks organizing the constant matrix elements whereas step six to thirteen represent the actual discrete time simulation over $M$ time steps, each of magnitude $\Delta t$. If the MEM is used instead of the CDM, then step seven and eleven are replaced by the associated MEM expressions for the velocity and the displacement, respectively, see e.g. [29]. Furthermore, the MEM acceleration should also be introduced in step seven. 


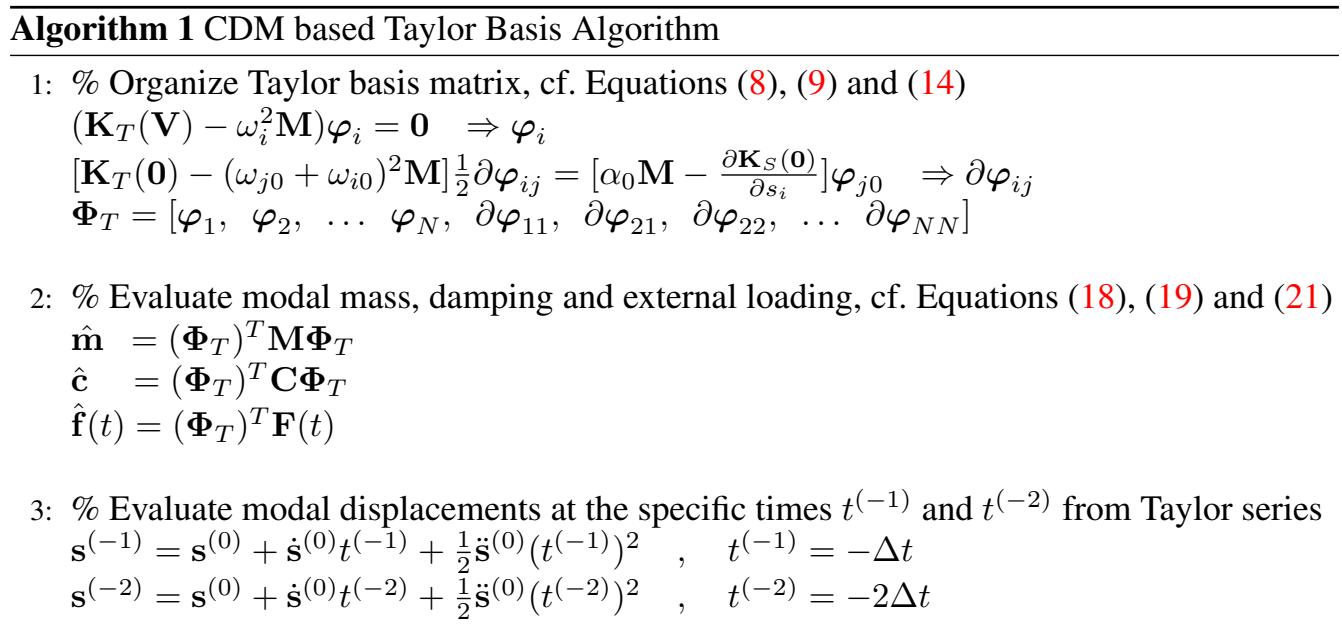

4: \% Evaluate constant equivalent force vectors, cf. Appendix C

$\overline{\mathbf{q}}_{1, i}, \overline{\mathbf{q}}_{2, i j}, \ldots, \overline{\mathbf{q}}_{6, i j k l m n}$

5: \% Evaluate transformation matrices, cf. Appendix A, Equations (49) and (59)

$\mathbf{H}, \mathbf{A}_{k}, \mathbf{B}_{k}$

6: \% Initiate simulation over $M$ time steps of magnitude $\Delta t$

for $q=0: M$

7: \% Evaluate velocity, cf. Equation (40)

$\dot{\mathbf{S}}^{(q)}=\frac{1}{\Delta t}\left(\frac{3}{2} \mathbf{S}^{(q)}-2 \mathbf{s}^{(q-1)}+\frac{1}{2} \mathbf{S}^{(q-2)}\right)$

8: \% Evaluate transformation matrices, cf. Appendix A, Equations (54) and (58)

$$
\begin{aligned}
& \mathbf{U}\left(\mathbf{s}^{(q)}\right)=\mathbf{H}+\sum_{k=1}^{N} \mathbf{B}_{k} s_{k}^{(q)} \\
& \mathbf{P}\left(\dot{\mathbf{s}}^{(q)}\right)=\sum_{k=1}^{N} \mathbf{A}_{k} \dot{s}_{k}^{(q)}
\end{aligned}
$$

9: \% Evaluate mass, damping and external loading, cf. Equations (30), (31) and (33)

$$
\begin{aligned}
\overline{\mathbf{m}}\left(\mathbf{s}^{(q)}\right) & =\mathbf{U}\left(\mathbf{s}^{(q)}\right)^{T} \hat{\mathbf{m}} \mathbf{U}\left(\mathbf{s}^{(q)}\right) \\
\overline{\mathbf{c}}\left(\mathbf{s}^{(q)}, \dot{\mathbf{s}}^{(q)}\right) & =\mathbf{U}\left(\mathbf{s}^{(q)}\right)^{T}\left(2 \hat{\mathbf{m}} \mathbf{P}\left(\dot{\mathbf{s}}^{(q)}\right)+\hat{\mathbf{c}} \mathbf{U}\left(\mathbf{s}^{(q)}\right)\right) \\
\overline{\mathbf{f}}\left(\mathbf{s}^{(q)}, t^{(q)}\right) & =\mathbf{U}\left(\mathbf{s}^{(q)}\right)^{T} \hat{\mathbf{f}}\left(t^{(q)}\right) \quad, \quad t^{(q)}=q \Delta t
\end{aligned}
$$

10: \% Evaluate internal restoring forces, cf. Equations (32) and (38)

$$
\begin{aligned}
\overline{\mathbf{g}}\left(\mathbf{s}^{(q)}\right) & =\mathbf{U}\left(\mathbf{s}^{(q)}\right)^{T} \hat{\mathbf{g}}\left(\mathbf{s}_{T}^{(q)}\right) \\
& =\mathbf{U}\left(\mathbf{s}^{(q)}\right)^{T}\left(\sum_{i=1}^{N} \overline{\mathbf{q}}_{1, i} s_{i}^{(q)}+\sum_{i=1}^{N} \sum_{j=1}^{i} \overline{\mathbf{q}}_{2, i j} s_{i}^{(q)} s_{j}^{(q)}+\ldots\right)
\end{aligned}
$$

11: \% Evaluate modal displacements by the CDM formula, cf. [30]

$$
\begin{gathered}
\mathbf{s}^{(q+1)}=\left(\frac{\overline{\mathbf{m}}\left(\mathbf{s}^{(q)}\right)}{\Delta t^{2}}+\frac{\overline{\mathbf{c}}\left(\mathbf{s}^{(q)}, \dot{\mathbf{s}}^{(q)}\right)}{2 \Delta t}\right)^{-1}\left(\overline{\mathbf{f}}\left(\mathbf{s}^{(q)}, t^{(q)}\right)-\overline{\mathbf{g}}\left(\mathbf{s}^{(q)}\right)+\frac{2 \overline{\mathbf{m}}\left(\mathbf{s}^{(q)}\right)}{\Delta t^{2}} \mathbf{s}^{(q)}-\right. \\
\left.\left[\frac{\overline{\mathbf{m}}\left(\mathbf{s}^{(q)}\right)}{\Delta t^{2}}-\frac{\overline{\mathbf{c}}\left(\mathbf{s}^{(q)}, \mathbf{s}^{(q)}\right)}{2 \Delta t}\right] \mathbf{s}^{(q-1)}\right)
\end{gathered}
$$

12: \% Transform modal solution into physical co-ordinates, cf. Equation (16)

$\mathbf{V}\left(\mathbf{s}^{(q+1)}\right)=\boldsymbol{\Phi}_{T} \mathbf{s}_{T}^{(q+1)}$

13: if $q=M$ end for 


\subsection{Case Validation of Nonlinear Frame}

In the following it is illustrated that the frame presented in Section 4.1 displays sufficient nonlinear behavior to be considered a relevant study case. For this the linear and nonlinear displacement responses of the frame are compared.

In Figure 2 the transverse displacements $u_{y}^{q}(t)$ and $u_{y}^{c}(t)$ are plotted as a function of time. Six displacement curves are shown; the linear Newmark response (LN), the nonlinear Newmark response $(\mathrm{NN})$, the linear CDM response (LC), the nonlinear CDM response (NC), the linear MEM response (LM) and the nonlinear MEM response (NM). All simulations are performed with the time step $\Delta t=55 \mu \mathrm{s}$, and a ten-second period is considered.

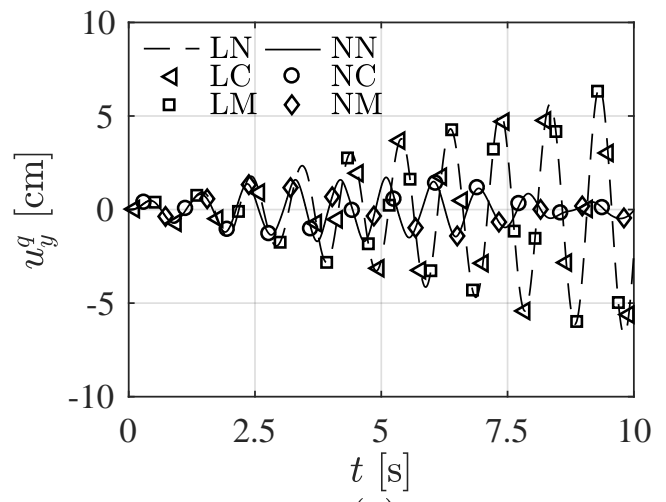

(a)

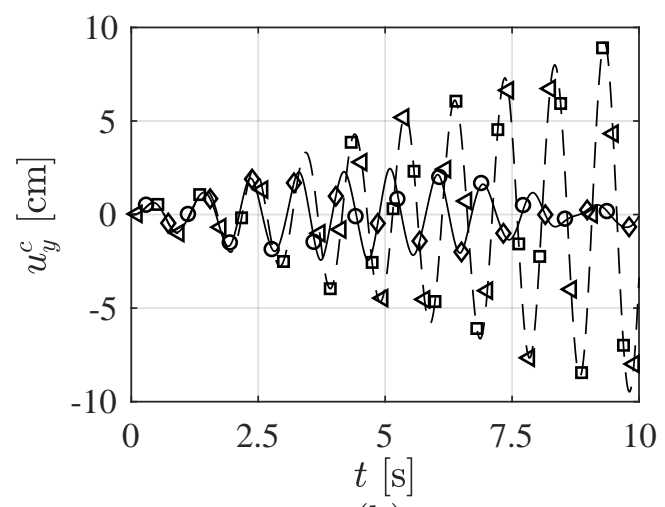

(b)

Figure 2. The (a) transverse quarter point displacement and the (b) transverse center point displacement of the horizontal frame part determined by the linear Newmark (LN), the linear CDM (LC), the linear MEM $(\mathrm{LM})$, the nonlinear Newmark (NN), the nonlinear CDM (NC) and the nonlinear MEM (NM) integration schemes.

From the two displacement plots in Figure 2 the response characteristics are seen to be the same; the linear response amplitudes appear to grow unboundedly, whereas the nonlinear response amplitudes are bounded, reaching their maximum after approximately five seconds. In addition to this the frequencies differ indicated by the fact that the nonlinear responses reach their local extrema before the linear solutions. Thus, altogether the nonlinear behavior of the system is very significant and the frame is considered a relevant study case.

Since the characteristics of the nonlinear displacements in the two points considered are the same, only the centerpoint displacement $u_{y}^{c}(t)$ will be considered in the following.

\subsection{Basis Analysis of the Nonlinear Frame}

In the present section the precision and the efficiency of the standard basis and the Taylor basis formulations are illustrated and compared by considering the nonlinear frame response.

In Figure 3 the first three frame mode shapes and their six associated MDs used in the basis simulations are plotted. The dashed lines mark the undeformed frame and the solid lines represent the basis vectors.

The three mode shapes in Figure 3 all represent pure bending modes, i.e. the displacements are only transverse to the element orientations, whereas the MDs contain both bending and axial displacements. In Part I it was illustrated that transverse displacements introduce axial displacements in kinematic nonlinear systems due to the formulation of the nonlinear strain measures. It is, therefore, expected that the MDs include axial displacements, as these represent the first order effects according to the Taylor series in (13).

In Figure 4 the frame response $u_{y}^{c}(t)$ is plotted as a function of time. Figure $4 \mathrm{a}$ considers a tensecond period and Figure $4 \mathrm{~b}$ a close-up. The response is evaluated by the Taylor basis referred to as $\boldsymbol{\Phi}_{T}^{N}$ and by the standard basis referred to as $\boldsymbol{\Phi}_{S}^{N+\bar{H}}$. The supercripts $N$ and $N+\bar{H}$ refer to the 

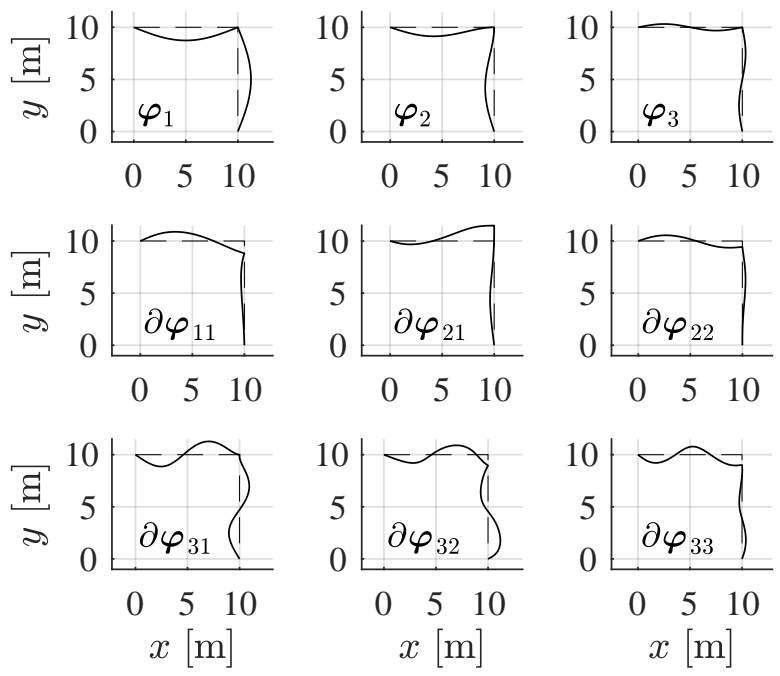

Figure 3. Mode shapes and MDs of the nonlinear frame.

number of free modal co-ordinates in the basis formulations; $N$ refers to the number of included mode shapes and $\bar{H}$ to the number of included MDs, $H$, minus the $P$ linear dependent basis vectors (i.e. $\bar{H}=H-P$ ). The Taylor basis also includes all of the $H$ MDs, i.e. the total number of basis vectors is $N+H$. However, the $H$ modal co-ordinates associated with the MDs are fixed by the Taylor series predictions. Therefore, they do not add to the number of free modal co-ordinates.

The basis responses shown in the plots are simulated using the CDM. Identical results are found with the MEM. The NC response presented in Figure 2 is also included in Figure 4. This is considered as the exact solution.

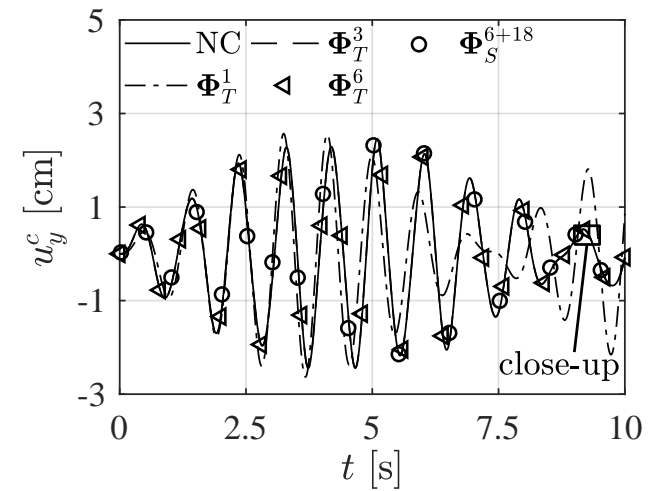

(a)

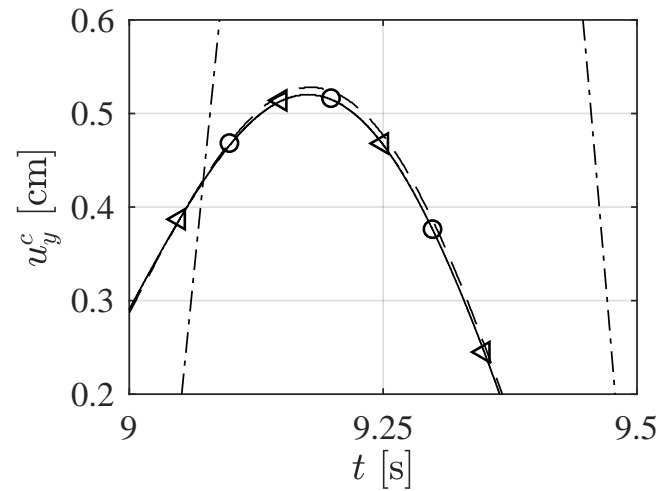

(b)

Figure 4. Transverse center displacement of the horizontal frame element considering (a) a 10 second interval and (b) a close-up. All responses are simulated by use of the CDM.

From Figure 4a the Taylor basis response for $N=1$ is seen to diverge significantly from the $\mathrm{NC}$ response. This is because more basis vectors are required to represent the nonlinear solution. However, the Taylor basis response is observed to rapidly converge towards the NC solution as the number of basis vectors increase. The close-up in Figure $4 \mathrm{~b}$ shows that the Taylor basis response is very close to the NC solution for $N=3$ basis vectors. For $N=6$ the difference is insignificant. The 
behavior of the standard basis is identical to the behavior of the Taylor basis for an equal number of mode shapes $N$. Therefore, only a single standard basis response with $N+\bar{H}=6+18$ basis vectors is plotted.

In Table II the time step magnitudes $\Delta t$ and the actual time $t_{\text {sim }}$ spent on simulating the ten-second responses shown in Figure 4 are listed. The table includes the simulation results based on the NC, the CDM and the MEM.

Table II. Simulation times and errors.

\begin{tabular}{c|c|ccc|ccc}
\hline & & $\Delta t$ & $t_{\text {sim }}$ & $e_{u}$ & $\Delta t^{\mathrm{lim}}$ & $t_{\text {sim }}^{\mathrm{lim}}$ & $e_{u}^{\mathrm{lim}}$ \\
Response & Algorithm & {$[\mathrm{ms}]$} & {$[\mathrm{s}]$} & {$[\%]$} & {$[\mathrm{ms}]$} & {$[\mathrm{s}]$} & {$[\%]$} \\
\hline $\mathrm{NC}$ & CDM & 0.055 & 276 & - & 0.055 & 276 & - \\
$\boldsymbol{\Phi}_{S}^{6+18}$ & CDM & 0.16 & 3.55 & 0.06 & 0.16 & 3.55 & 0.06 \\
$\boldsymbol{\Phi}_{T}^{6}$ & CDM & 2.00 & 0.25 & 0.10 & 28.9 & 0.03 & 5.21 \\
\hline $\boldsymbol{\Phi}_{S}^{6+18}$ & MEM & 0.16 & 3.57 & 0.06 & 0.16 & 3.57 & 0.06 \\
$\boldsymbol{\Phi}_{T}^{6}$ & MEM & 2.00 & 0.32 & 0.10 & 28.9 & 0.03 & 5.21 \\
\hline
\end{tabular}

A relative difference between the $\mathrm{NC}$ response and the basis responses is also presented in Table II as an error $e_{u}$ which is defined as

$$
e_{u}=\left(\frac{\left|u_{y, \mathrm{NC}}^{c}(t)-u_{y, \text { basis }}^{c}(t)\right|}{\left|u_{y, \mathrm{NC}}^{c}(t)\right|_{\max }}\right)_{\text {max }} \quad, \quad t \in[0 ; 10 \mathrm{~s}]
$$

where the subscript 'NC' refers to the NC solution, 'basis' refers to either the Taylor basis or the standard basis and 'max' refers to the maximum value in the considered time interval. In Table II the limit values for the time step $\Delta t^{\mathrm{lim}}$ are also listed, defined as the maximum values that can be used before instability occurs. Instability is characterized by sudden rapid fluctuations or amplitudes increasing without apparent limit within a short time interval. The corresponding simulation times and errors are denoted $t_{\text {sim }}^{\lim }$ and $e_{u}^{\lim }$.

From Table II the time step magnitude used in the NC simulation in Figure 4 is seen to be $55 \mu$ s with a simulation time of $276 \mathrm{~s}$. Both of the listed standard basis responses are simulated with a time step of $0.16 \mathrm{~ms}$ producing errors of $0.06 \%$. The simulation times are almost identical independent of the integration scheme; with the CDM the simulation is $3.55 \mathrm{~s}$ and with MEM the simulation time is $3.57 \mathrm{~s}$. In the Taylor basis simulations the time step applied is $2 \mathrm{~ms}$ with an error of $0.10 \%$. The Taylor basis simulation time using the CDM is $0.25 \mathrm{~s}$. With the MEM the Taylor basis simulation time increases to $0.32 \mathrm{~s}$. Thus, independent of the explicit algorithm, the Taylor basis simulation times are one order of magnitude smaller than those obtained with the standard basis and more than a thousand times smaller than those obtained with the $\mathrm{NC}$ algortihm in the actual case.

The time steps $\Delta t$ listed in Table II that are used for the NC simulations and the standard basis simulations are also the limit values. However, in the case of the Taylor basis, the $2 \mathrm{~ms}$ time step magnitude can be further increased. As stated in the table, the limit time step value is $28.90 \mathrm{~s}$ for both the CDM and the MEM with a simulation time of $0.03 \mathrm{~s}$ and a relative error of $5.21 \%$. Thus, the Taylor basis exhibits significantly higher stability than the standard basis.

In Figure 5 the relation between the errors, the simulation times and the time step magnitudes are presented. The plot illustrates the error $e_{u}$ as a function of the simulation time $t_{\text {sim }}$ using the Taylor basis and the standard basis simulated using the CDM. The Taylor basis is illustrated for $N=6$ and the standard basis is illustrated for $N+\bar{H}=3+6$ and $N+\bar{H}=6+18$. The applied time steps $\Delta t$ are indicated next to each discrete point in brackets, with the limit values marked by an asterisk $(*)$.

The plot in Figure 5 shows that if the time step is decreased the Taylor basis error is increased simultaneously whereas the simulation time is decreased. The limit time steps is $28.9 \mathrm{~ms}$ with an error of $e_{u}^{\lim }=5.21 \%$. As the simulation time grows the error converges to a value of approximately $e_{u}=0.08 \%$. Both curves representing the standard basis display a more or less constant error level 


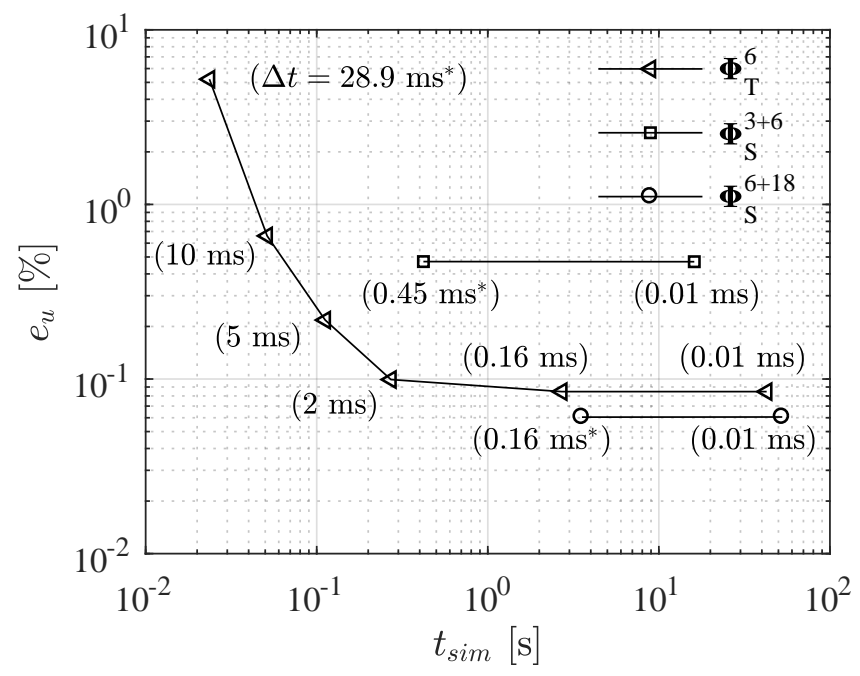

Figure 5. The error $e_{u}$ as a function of the simulation time $t_{\text {sim }}$ of the ten-second frame response evaluated by basis projection based on the CDM. Time step magnitudes $\Delta t$ are added in brackets with $\left(^{*}\right)$ indicating the limit values.

for all time steps. The converged error values are $e_{u}=0.47 \%$ and $e_{u}=0.06 \%$, respectively, of which the lowest value represents the basis with $N+\bar{H}=6+18$ basis vectors.

The nonzero converged errors in Figure 5 indicate that additional modes are required to reach a full response convergence. The small difference in the converged Taylor basis error for $N=6$ and the converged standard basis error for $N+\bar{H}=6+18$ is explained by the fact, that the standard basis has $N+\bar{H}=6+18=24$ free modal co-ordinates, whereas the Taylor basis only has $N=6$ free modal co-ordinates.

Based on the results in Table II and in Figure 5 the Taylor basis is concluded to be the preferred basis when considering a combination of precision and computational efficiency. The argument for this is, that it is possible to perform simulations significantly faster with the Taylor basis compared to the standard basis, with errors in the same order of magnitude or even smaller.

\subsection{Basis Stability}

The stability of the Taylor basis and the standard basis are studied. As a measure for the stability it is considered how large time steps $\Delta t$ that can be applied as a function of the basis vector frequencies $\omega$ without introducing sudden rapid fluctuations or significant increases in the response amplitude.

In Figure 6 the maximum time step magnitudes that can be used in the simulations of the frame response are plotted using, respectively, the Taylor basis, $\Delta \mathrm{t}_{T}$, and the standard basis, $\Delta \mathrm{t}_{S}$. The results are based on the CDM; however, identical results are obtained with the MEM.

In Figure $6 \mathrm{a}$ the time step $\Delta \mathrm{t}_{T}$ is plotted as a function of the frequency $\omega_{\varphi}$ and in Figure $6 \mathrm{~b}$ the time step $\Delta \mathrm{t}_{S}$ is plotted as a function of the frequency $\omega_{\partial \varphi}$. The former frequency refers to the highest frequency associated with the mode shapes of the system, and the latter refers to the highest frequency associated with the MDs of the system. These are approximated by Rayleigh's quotient as

$$
\begin{aligned}
\omega_{\varphi}^{2} & =\left(\frac{\boldsymbol{\varphi}_{i}^{T} \mathbf{K}_{T}(\mathbf{0}) \boldsymbol{\varphi}_{i}}{\boldsymbol{\varphi}_{i}^{T} \mathbf{M} \boldsymbol{\varphi}_{i}}\right)_{\text {max }}, i \epsilon[1 ; N] \\
\omega_{\partial \varphi}^{2} & =\left(\frac{\partial \boldsymbol{\varphi}_{i j}^{T} \mathbf{K}_{T}(\mathbf{0}) \partial \boldsymbol{\varphi}_{i j}}{\partial \boldsymbol{\varphi}_{i j}^{T} \mathbf{M} \partial \boldsymbol{\varphi}_{i j}}\right)_{\max } \quad, i, j \epsilon[1 ; N] \wedge j \leq i
\end{aligned}
$$

For simplicity, only the first mode shape $\varphi_{1}$ and its associated $\operatorname{MD} \partial \varphi_{11}$ are included in the Taylor basis used to generate the results in Figure 6. Two analytical stability limits, $\Delta \mathrm{t}_{\varphi}$ and $\Delta \mathrm{t}_{\partial \varphi}$, are also 


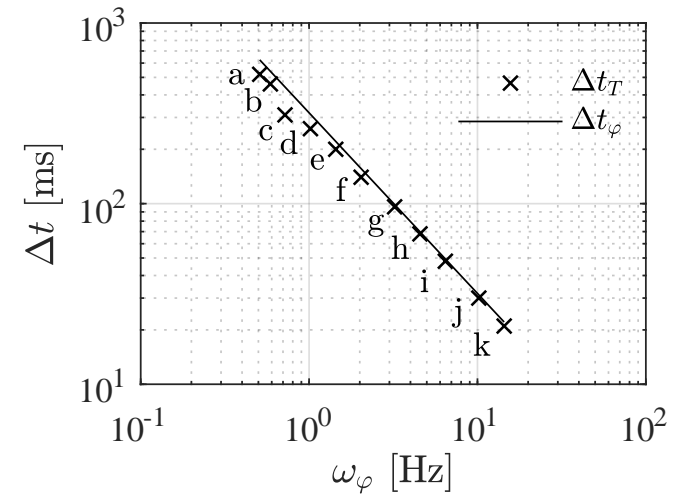

(a)

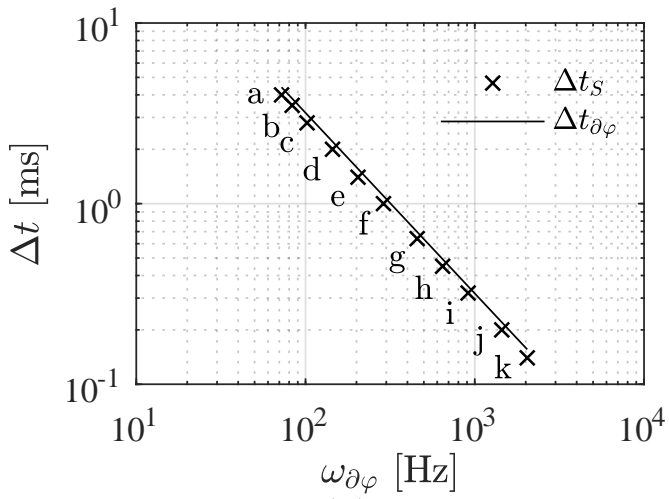

(b)

Figure 6. Theoretical CDM time step stability curves, $\Delta t_{\varphi}$ and $\Delta t_{\partial \varphi}$, compared with the stability of (a) the Taylor basis $\Delta t_{T}$ and (b) the standard basis $\Delta t_{S}$. The marks a to k refer to different frame density states.

plotted. These limits are found by inserting, respectively, the mode shape frequency and the MD frequency into the stability condition for linear systems simulated by the CDM and the MEM, see e.g. $[29,31]$ :

$$
\Delta t_{\varphi}=\frac{2}{\omega_{\varphi}}, \Delta t_{\partial \varphi}=\frac{2}{\omega_{\partial \varphi}}
$$

To investigate the basis stabilities for a wide range of frequencies, the frame density, $\rho$, was scaled during the analysis. Each mark ranging from a to $\mathrm{k}$ in Figure 6 represents a specific density.

From Figure 6a the Taylor basis limit time steps, $\Delta \mathrm{t}_{T}$, are seen to be very close to the analytical stability curve, $\Delta t_{\varphi}$. The result points $\mathrm{g}$ to $\mathrm{k}$ only deviate by a maximum of 10 percent from the analytical $\Delta t_{\varphi}$ curve. Considering the result points a to $\mathrm{j}$ the deviation is slightly larger, with a maximum deviation of 30 percent in point c. The standard basis time steps, $\Delta \mathrm{t}_{S}$, shown in the plot in Figure $6 b$, are also seen to be located closely to the analytical time step curve $\Delta t_{\partial \varphi}$. The relative deviation between the discrete result points and the analytical time step curve is in the 8-10 percent interval for the frequency domain considered.

The above reported observations indicate that the stability of the Taylor basis only depends on the mode shapes frequencies, whereas the stability of the standard basis depends on the frequencies associated with the MDs. Thus, an approximate magnitude for the ratio between the limit time step magnitudes is given as the ratio between the two analytical stability limits in (44), i.e.:

$$
\frac{\Delta t_{T}^{\lim }}{\Delta t_{S}^{\lim }} \approx \frac{\Delta t_{\varphi}}{\Delta t_{\partial \varphi}}=\frac{\omega_{\partial \varphi}}{\omega_{\varphi}}
$$

As indicated by the magnitudes of the frequencies in Figure 6, it is not unlikely that $\omega_{\partial \varphi} \gg \omega_{\varphi}$ for an arbitrary density state. Thus, potentially the time steps applied with the Taylor basis can be significantly increased compared to those used in the standard basis. In the case illustrated in Figure 4 the highest frequency associated with the included mode shapes relates to the basis vector $\varphi_{6}$ and is $\omega_{6}=68 \mathrm{rad} / \mathrm{s}$. According to (44) this corresponds to a time step stability limit value

$$
\Delta t_{\varphi}=\frac{2}{\omega_{6}}=29.58 \mathrm{~ms}
$$

The time step value in (46) is close to the stability limit for the Taylor basis reported in Table II which is $\Delta t^{\mathrm{lim}}=28.9 \mathrm{~ms}$. The highest frequency associated with the MDs included in the Figure 4 simulations relates to the basis vector $\partial \varphi_{61}$ and is $\omega_{61}=3463 \mathrm{rad} / \mathrm{s}$. According to (44) this 
corresponds to a time step stability limit of

$$
\Delta t_{\partial \varphi}=\frac{2}{\omega_{61}}=0.58 \mathrm{~ms}
$$

Given the time step values in (46) and (47) the approximate ratio in (45) is

$$
\frac{\Delta t_{T}}{\Delta t_{S}} \approx \frac{29.58 \mathrm{~ms}}{0.58 \mathrm{~ms}}=51
$$

The ratio of the actual time step limits in Table II is $28.9 \mathrm{~ms} / 0.16 \mathrm{~ms} \approx 180$, which is significantly higher than the predicted time step ratio in (48). Thus, in the given case the approximation in (45) is a lower bound value.

\section{CONCLUSION}

In the present work the efficiency, stability and accuracy of a Taylor basis used to analyse kinematically nonlinear systems was investigated. The advantage of projecting the discretized equations of motion onto a Taylor basis is that the co-ordinates of the modal derivatives are given as a fixed product of the system mode shape co-ordinates. Thus, a Taylor basis that includes $N$ system mode shapes and $H=\frac{1}{2}\left(N+N^{2}\right)$ modal derivatives contains only $N$ unknowns to be determined. Considering the response of a highly nonlinear frame, it was shown that the Taylor basis exhibits high precision. The example also showed that the computational time can be reduced by one order of magnitude by using a Taylor basis instead of a standard basis including the same basis vectors. Furthermore, it was illustrated that the Taylor basis allows for significantly higher time steps to be used before instability occurs than the standard basis does. The ratio between the maximum allowable time steps of the Taylor basis and the standard basis was shown to be approximately equal to the frequencies of the modal derivatives divided by the mode shape frequencies. Since the frequencies of the modal derivatives are typically significantly higher than the mode shapes frequencies, this potentially allows for a significantly higher time step to be applied with a Taylor basis.

\section{A. TRANSFORMATION OF THE TAYLOR CO-ORDINATES}

In the following the matrices used in the transformation of the EOMs in (17) from the (s $\mathbf{s}_{T}, \dot{\mathbf{s}}_{T}, \ddot{\mathbf{s}}_{T}$ ) co-ordinates to the $(\mathbf{s}, \dot{\mathbf{s}}, \ddot{\mathbf{s}})$ co-ordinates are presented. For the purpose the two matrices $\mathbf{H}$ and $\mathbf{E}_{i j}$ are introduced:

$$
\mathbf{H}=\left[\begin{array}{c}
\mathbf{I} \\
\mathbf{0}_{1}
\end{array}\right], \quad \mathbf{E}_{i j}=\mathbf{E}_{j i}^{T}=\left[\begin{array}{cccc}
\delta_{1 i} \delta_{j 1} & \delta_{1 i} \delta_{j 2} & \ldots & \delta_{1 i} \delta_{j N} \\
\delta_{2 i} \delta_{j 1} & \delta_{2 i} \delta_{j 2} & \ldots & \delta_{2 i} \delta_{j N} \\
\vdots & \vdots & & \vdots \\
\delta_{N i} \delta_{j 1} & \delta_{N i} \delta_{j 2} & \ldots & \delta_{N i} \delta_{j N}
\end{array}\right]
$$

with $\mathbf{I}$ being a unit matrix of dimension $N \times N, \mathbf{0}_{1}$ a zero matrix of dimension $H \times N$ and $\mathbf{E}_{i j}$ a $N \times N$ matrix. This produces a matrix $\mathbf{H}$ of dimension $(N+H) \times H$. Furthermore, the vectors $\mathbf{e}_{i j}$ and $\mathbf{d}_{i j}$ are introduced

$$
\mathbf{e}_{i j}=\left\{\begin{array}{c}
\mathbf{0}_{2} \\
\mathbf{d}_{i j}
\end{array}\right\}, \quad \mathbf{d}_{i j}=\left[\begin{array}{lllllll}
\delta_{1 i} \delta_{j 1} & \delta_{2 i} \delta_{j 1} & \delta_{2 i} \delta_{j 2} & \delta_{3 i} \delta_{j 1} & \delta_{3 i} \delta_{j 2} & \ldots & \delta_{N i} \delta_{j N}
\end{array}\right]^{T}
$$

where $\mathbf{d}_{i j}$ is a vector of dimension $H \times 1, \mathbf{0}_{2}$ is a zero vector of dimension $N \times 1$ and $\mathbf{e}_{i j}$ is a vector of dimension $(H+N) \times 1$. Given these defined vectors and matrices, the relation between $\mathbf{s}_{T}$ and $\mathbf{s}$ can be written as

$$
\mathbf{s}_{T}=\left(\mathbf{H}+\sum_{i=1}^{N} \sum_{j=1}^{i} \mathbf{e}_{i j} \mathbf{s}^{T} \mathbf{E}_{i j}\right) \mathbf{s}=(\mathbf{H}+\mathbf{W}(\mathbf{s})) \mathbf{s}=\mathbf{Q}(\mathbf{s}) \mathbf{s}
$$


where

$$
\mathbf{Q}(\mathbf{s})=\mathbf{H}+\mathbf{W}(\mathbf{s}), \quad \mathbf{W}(\mathbf{s})=\sum_{i=1}^{N} \sum_{j=1}^{i} \mathbf{e}_{i j} \mathbf{s}^{T} \mathbf{E}_{i j}
$$

Furthermore, the relation between $\dot{\mathbf{s}}_{T}$ and the $(\mathbf{s}, \dot{\mathbf{s}}, \ddot{\mathbf{s}})$ co-ordinates is

$$
\dot{\mathbf{s}}_{T}=\left(\mathbf{H}+\sum_{i=1}^{N} \sum_{j=1}^{i} \mathbf{e}_{i j} \mathbf{s}^{T}\left(\mathbf{E}_{i j}+\mathbf{E}_{j i}\right)\right) \dot{\mathbf{s}}=(\mathbf{H}+\mathbf{R}(\mathbf{s})) \dot{\mathbf{s}}=\mathbf{U}(\mathbf{s}) \dot{\mathbf{s}}
$$

where

$$
\mathbf{U}(\mathbf{s})=\mathbf{H}+\mathbf{R}(\mathbf{s}) \quad, \quad \mathbf{R}(\mathbf{s})=\sum_{i=1}^{N} \sum_{j=1}^{i} \mathbf{e}_{i j} \mathbf{s}^{T}\left(\mathbf{E}_{i j}+\mathbf{E}_{j i}\right)
$$

The $\ddot{\mathbf{s}}_{T}$ vector is related to the $(\mathbf{s}, \dot{\mathbf{s}}, \ddot{\mathbf{s}})$ co-ordinates through the relation

$$
\ddot{\mathbf{s}}_{T}=\mathbf{U}(\mathbf{s}) \ddot{\mathbf{s}}+2 \mathbf{P}(\dot{\mathbf{s}}) \dot{\mathbf{s}} \quad, \quad \mathbf{P}(\dot{\mathbf{s}})=\sum_{i=1}^{N} \sum_{j=1}^{i} \mathbf{e}_{i j} \dot{\mathbf{s}}^{T} \mathbf{E}_{i j}
$$

Finally the variation $\delta \mathbf{s}_{T}$ can be related to $\delta$ s by use of the expression

$$
\delta \mathbf{s}_{T}=\mathbf{U}(\mathbf{s}) \delta \mathbf{s}
$$

The vector $\mathbf{s}$ is now introduced in a sum format written in the following manner

$$
\mathbf{s}=\sum_{k=1}^{N} \mathbf{h}_{k} s_{k}, \quad \mathbf{h}_{k}^{T}=\left[\begin{array}{llll}
\delta_{1 k} & \delta_{2 k} & \ldots & \delta_{N k}
\end{array}\right]
$$

Introducing the sum formulation in (57) into the definitions for the matrices $\mathbf{W}(\mathbf{s}), \mathbf{R}(\mathbf{s})$ and $\mathbf{P}(\dot{\mathbf{s}})$ enables them to be written in a more compact form as

$$
\mathbf{W}(\mathbf{s})=\sum_{k=1}^{N} \mathbf{A}_{k} s_{k}, \quad \mathbf{R}(\mathbf{s})=\sum_{k=1}^{N} \mathbf{B}_{k} s_{k}, \quad \mathbf{P}(\dot{\mathbf{s}})=\sum_{k=1}^{N} \mathbf{A}_{k} \dot{s}_{k}
$$

with the constant matrices

$$
\mathbf{A}_{k}=\sum_{i=1}^{N} \sum_{j=1}^{i} \mathbf{e}_{i j} \mathbf{h}_{k}^{T} \mathbf{E}_{i j}, \quad \mathbf{B}_{k}=\sum_{i=1}^{N} \sum_{j=1}^{i} \mathbf{e}_{i j} \mathbf{h}_{k}^{T}\left(\mathbf{E}_{i j}+\mathbf{E}_{j i}\right)
$$

\section{B. POLYNOMIAL INTERNAL RESTORING FORCE VECTORS - THE STANDARD BASIS}

In the following it is illustrated how the element local internal restoring forces projected onto a standard basis can be formulated as vector polynomial. In kinematic nonlinear systems the internal restoring forces in (36) of an element covering the domain $\Omega$ can be written in the general form as, see e.g. [32];

$$
\begin{aligned}
\mathbf{g}_{e}(\mathbf{v})= & {[\underbrace{\int_{\Omega} \mathbf{B}^{T} \mathbf{D B} d \Omega}_{\mathbf{K}_{0}}+\underbrace{\frac{1}{2} \int_{\Omega} \sum_{l=1}^{\mu}\left(\mathbf{B}^{T} \mathbf{D} \mathbf{I}_{l} \mathbf{v}^{T} \mathbf{S}_{l}+2 \mathbf{S}_{l} \mathbf{v} \mathbf{I}_{l} \mathbf{D B}\right) d \Omega}_{\mathbf{K}_{1}(\mathbf{v})}} \\
& +\underbrace{\frac{1}{2} \int_{\Omega} \sum_{l=1}^{\mu} \sum_{m=1}^{\mu} \mathbf{S}_{l} \mathbf{v} \mathbf{I}_{l}^{T} \mathbf{D} \mathbf{I}_{m} \mathbf{v}^{T} \mathbf{S}_{m} d \Omega}_{\mathbf{K}_{2}(\mathbf{v})}] \mathbf{v}
\end{aligned}
$$


where the subindex $e$ refers to the specific element number and $\mathbf{v}$ contains the element local DOFs. Furthermore, $\mathbf{B}$ is a matrix from linear formulation, $\mathbf{S}_{\ell}$ is a symmetric matrix originating from nonlinear strain contributions, $\mathbf{D}$ is a stiffness matrix, $\mathbf{I}_{\ell}$ is a column vector which places the nonlinear terms in the correct position and $\mu$ is the number of nonlinear strain terms included. By multiplying the vectors $\mathbf{K}_{0} \mathbf{v}, \mathbf{K}_{1}(\mathbf{v}) \mathbf{v}$ and $\mathbf{K}_{2}(\mathbf{v}) \mathbf{v}$ in (60) by the element local basis matrix $\boldsymbol{\Phi}_{e}^{T}$ on the left-hand side and inserting the relation $\mathbf{v}=\Phi_{e} \mathbf{s}$, the vector terms can be expressed as three summations of vectors multiplied by the reduced co-ordinates:

$$
\begin{aligned}
\boldsymbol{\Phi}_{e}^{T} \mathbf{K}_{0} \boldsymbol{\Phi}_{e} \mathbf{s} & =\sum_{i=1}^{R} \mathbf{q}_{1, i} s_{i} \\
\boldsymbol{\Phi}_{e}^{T} \mathbf{K}_{1}\left(\boldsymbol{\Phi}_{e} \mathbf{s}\right) \boldsymbol{\Phi}_{e} \mathbf{s} & =\sum_{i=1}^{R} \sum_{j=1}^{R} \tilde{\mathbf{q}}_{2, i j} s_{i} s_{j}=\sum_{i=1}^{R} \sum_{j=1}^{i} \mathbf{q}_{2, i j} s_{i} s_{j} \\
\boldsymbol{\Phi}_{e}^{T} \mathbf{K}_{2}\left(\boldsymbol{\Phi}_{e} \mathbf{s}\right) \boldsymbol{\Phi}_{e} \mathbf{s} & =\sum_{i=1}^{R} \sum_{j=1}^{R} \sum_{k=1}^{R} \tilde{\mathbf{q}}_{3, i j k} s_{i} s_{j} s_{k}=\sum_{i=1}^{R} \sum_{j=1}^{i} \sum_{k=1}^{j} \mathbf{q}_{3, i j k} s_{i} s_{j} s_{k}
\end{aligned}
$$

The terms $\mathbf{q}_{1, i}, \mathbf{q}_{2, i j}, \mathbf{q}_{3, i j k}, \tilde{\mathbf{q}}_{2, i j}$ and $\tilde{\mathbf{q}}_{3, i j k}$ are constant equivalent force vectors, and $R=N+\bar{H}$ represents the $N$ mode shapes plus the $\bar{H}$ linear independent MDs included in the standard basis. The vectors $\mathbf{q}_{2, i j}$ and $\mathbf{q}_{3, i j k}$ represent a compact formulation of the vectors $\tilde{\mathbf{q}}_{2, i j}$ and $\tilde{\mathbf{q}}_{3, i j k}$. The relations between the vectors are

$$
\begin{aligned}
\mathbf{q}_{2, i j} & =\tilde{\mathbf{q}}_{2, i j}+\left(1-\delta_{i j}\right) \tilde{\mathbf{q}}_{2, j i} \\
\mathbf{q}_{3, i j k} & =\tilde{\mathbf{q}}_{3, i j k}+\left(\tilde{\mathbf{q}}_{3, j k i}+\tilde{\mathbf{q}}_{3, k i j}\right)\left(1-\delta_{i j} \delta_{j k}\right)-\left(\tilde{\mathbf{q}}_{3, i k j}+\tilde{\mathbf{q}}_{3, k j i}+\tilde{\mathbf{q}}_{3, j i k}\right) \varepsilon_{i j k}
\end{aligned}
$$

where $\varepsilon_{i j k}$ is a permutation symbol fulfilling the conditions

$$
\begin{aligned}
& \varepsilon_{i j k}=0 \text { if } i=j \vee i=k \vee j=k \\
& \varepsilon_{i j k}=1 \text { if }(i, j, k)=(1,2,3) \vee(2,3,1) \vee(3,1,2) \\
& \varepsilon_{i j k}=-1 \text { if }(i, j, k)=(3,2,1) \vee(2,1,3) \vee(1,3,2)
\end{aligned}
$$

If vector number $i$ in the element local basis matrix $\boldsymbol{\Phi}_{e}$ is denoted as $\boldsymbol{\Phi}_{e, i}$, then the three vectors $\mathbf{q}_{1, i}, \tilde{\mathbf{q}}_{2, i j}$ and $\tilde{\mathbf{q}}_{3, i j k}$ are given as

$$
\begin{aligned}
\mathbf{q}_{1, i} & =\boldsymbol{\Phi}_{e}^{T} \mathbf{K}_{0} \boldsymbol{\Phi}_{e, i}=\boldsymbol{\Phi}_{e}^{T}\left(\int_{\Omega} \mathbf{B}^{T} \mathbf{D B} d \Omega\right) \boldsymbol{\Phi}_{e, i} \\
\tilde{\mathbf{q}}_{2, i j} & =\boldsymbol{\Phi}_{e}^{T} \mathbf{K}_{1}\left(\boldsymbol{\Phi}_{e, i}\right) \boldsymbol{\Phi}_{e, j}=\boldsymbol{\Phi}_{e}^{T}\left(\frac{1}{2} \int_{\Omega} \sum_{l=1}^{r}\left(\mathbf{B}^{T} \mathbf{D} \mathbf{I}_{l} \boldsymbol{\Phi}_{e, i}^{T} \mathbf{S}_{l}+2 \mathbf{S}_{l} \boldsymbol{\Phi}_{e, i} \mathbf{I}_{l} \mathbf{D B}\right) d \Omega\right) \boldsymbol{\Phi}_{e, j} \\
\tilde{\mathbf{q}}_{3, i j k} & =\boldsymbol{\Phi}_{e}^{T} \overline{\mathbf{K}}_{2}\left(\boldsymbol{\Phi}_{e, i}, \boldsymbol{\Phi}_{e, j}\right) \boldsymbol{\Phi}_{e, k}=\boldsymbol{\Phi}_{e}^{T}\left(\frac{1}{2} \int_{\Omega} \sum_{l=1}^{r} \sum_{m=1}^{r} \mathbf{S}_{l} \boldsymbol{\Phi}_{e, i} \mathbf{I}_{l}^{T} \mathbf{D} \mathbf{I}_{m} \boldsymbol{\Phi}_{e, j}^{T} \mathbf{S}_{m} d \Omega\right) \boldsymbol{\Phi}_{e, k}
\end{aligned}
$$

In (68) the following notation has been introduced

$$
\mathbf{K}_{2}(\mathbf{v})=\overline{\mathbf{K}}_{2}\left(\mathbf{v}_{1}, \mathbf{v}_{2}\right)
$$

where the subindeces 1 and 2 refer to, respectively, the first and second element local displacement vector from the left in $\mathbf{K}_{2}(\mathbf{v})$. By inserting the expressions in (61)-(63) into (60), the element local projected internal restoring forces can be written in the compact format

$$
\begin{aligned}
\tilde{\mathbf{g}}_{e}(\mathbf{s}) & =\boldsymbol{\Phi}_{e}^{T}\left(\mathbf{K}_{0}+\mathbf{K}_{1}\left(\boldsymbol{\Phi}_{e} \mathbf{s}\right)+\mathbf{K}_{2}\left(\boldsymbol{\Phi}_{e} \mathbf{s}\right)\right) \boldsymbol{\Phi}_{e} \mathbf{s} \\
& =\sum_{i=1}^{R} \mathbf{q}_{1, i} s_{i}+\sum_{i=1}^{R} \sum_{j=1}^{i} \mathbf{q}_{2, i j} s_{i} s_{j}+\sum_{i=1}^{R} \sum_{j=1}^{i} \sum_{k=1}^{j} \mathbf{q}_{3, i j k} s_{i} s_{j} s_{k}
\end{aligned}
$$

The equivalent force vector coefficients in (70) represent element local contributions. To obtain the global equivalent force vector coefficients, all local element contributions in (70) are assembled. 


\section{POLYNOMIAL INTERNAL RESTORING FORCE VECTORS - THE TAYLOR BASIS}

In the following it is explained how the element local internal restoring forces projected onto a Taylor basis can be formulated as a vector polynomial. This is done by first replacing the element local DOFs in (60) with the Taylor basis formulation $\mathbf{v}=\boldsymbol{\Phi}_{T, e} \mathbf{s}_{T}$. After this the element local internal restoring forces are multiplied by the element local Taylor basis matrix $\boldsymbol{\Phi}_{T, e}^{T}$ on the left hand side and organized as a sixth order vector polynomial in the reduced co-ordinates as

$$
\begin{aligned}
\hat{\mathbf{g}}_{e}\left(\mathbf{s}_{T}\right) & =\boldsymbol{\Phi}_{T, e}^{T}\left(\mathbf{K}_{0}+\mathbf{K}_{1}\left(\mathbf{\Phi}_{T, e} \mathbf{s}_{T}\right)+\mathbf{K}_{2}\left(\boldsymbol{\Phi}_{T, e} \mathbf{s}_{T}\right)\right) \boldsymbol{\Phi}_{T, e} \mathbf{s}_{T} \\
& =\sum_{i=1}^{N} \mathbf{q}_{1, i}^{*} s_{i}+\sum_{i=1}^{N} \sum_{j=1}^{N} \mathbf{q}_{2, i j}^{*} s_{i} s_{j}+\cdots+\sum_{i=1}^{N} \sum_{j=1}^{N} \sum_{k=1}^{N} \sum_{l=1}^{N} \sum_{m=1}^{N} \sum_{n=1}^{N} \mathbf{q}_{6, i j k l m n}^{*} s_{i} s_{j} s_{k} s_{l} s_{m} s_{n} \\
& =\sum_{i=1}^{N} \mathbf{q}_{1, i}^{*} s_{i}+\sum_{i=1}^{N} \sum_{j=1}^{i} \overline{\mathbf{q}}_{2, i j} s_{i} s_{j}+\cdots+\sum_{i=1}^{N} \sum_{j=1}^{i} \sum_{k=1}^{j} \sum_{l=1}^{k} \sum_{m=1}^{l} \sum_{n=1}^{m} \overline{\mathbf{q}}_{6, i j k l m n} s_{i} s_{j} s_{k} s_{l} s_{m} s_{n}
\end{aligned}
$$

where $\mathbf{q}_{1, i}^{*}, \mathbf{q}_{2, i j}^{*}, \ldots, \mathbf{q}_{6, i j k l m n}^{*}, \overline{\mathbf{q}}_{2, i j}, \overline{\mathbf{q}}_{3, i j k}, \ldots$ and $\overline{\mathbf{q}}_{6, i j k l m n}$ are all constant vector coefficients. The coefficients $\overline{\mathbf{q}}$ represent a compact formulation of the vectors coeffcients $\mathbf{q}$. These compact vector coefficients are found in two steps. First the vectors $\mathbf{q}^{*}$ are organized. Using the definition in (69) these are given as

$$
\begin{aligned}
\mathbf{q}_{1, i}^{*}= & \left(\boldsymbol{\Phi}_{T, e}\right)^{T} \mathbf{K}_{0} \boldsymbol{\varphi}_{i, e} \\
\mathbf{q}_{2, i j}^{*}= & \left(\boldsymbol{\Phi}_{T, e}\right)^{T}\left(\mathbf{K}_{0} \partial \boldsymbol{\varphi}_{i j, e}+\mathbf{K}_{1}\left(\boldsymbol{\varphi}_{i, e}\right) \boldsymbol{\varphi}_{j, e}\right) \\
\mathbf{q}_{3, i j k}^{*}= & \left(\boldsymbol{\Phi}_{T, e}\right)^{T}\left(\mathbf{K}_{1}\left(\boldsymbol{\varphi}_{i, e}\right) \partial \boldsymbol{\varphi}_{j k, e}+\mathbf{K}_{1}\left(\partial \boldsymbol{\varphi}_{i j, e}\right) \boldsymbol{\varphi}_{k, e}+\overline{\mathbf{K}}_{2}\left(\boldsymbol{\varphi}_{i, e}, \boldsymbol{\varphi}_{j, e}\right) \boldsymbol{\varphi}_{k, e}\right) \\
\mathbf{q}_{4, i j k l}= & \left(\boldsymbol{\Phi}_{T, e}\right)^{T}\left(\mathbf{K}_{1}\left(\partial \boldsymbol{\varphi}_{i j, e}\right) \partial \boldsymbol{\varphi}_{k l, e}+\overline{\mathbf{K}}_{2}\left(\boldsymbol{\varphi}_{i, e}, \boldsymbol{\varphi}_{j, e}\right) \partial \boldsymbol{\varphi}_{k l, e}+\right. \\
\left.\overline{\mathbf{K}}_{2}\left(\partial \boldsymbol{\varphi}_{i j, e}, \boldsymbol{\varphi}_{k, e}\right) \boldsymbol{\varphi}_{l, e}+\overline{\mathbf{K}}_{2}\left(\boldsymbol{\varphi}_{i, e}, \partial \boldsymbol{\varphi}_{j k, e}\right) \boldsymbol{\varphi}_{l, e}\right) & \\
\mathbf{q}_{5, i j k l m}^{*}= & \left(\boldsymbol{\Phi}_{T, e}\right)^{T}\left(\overline{\mathbf{K}}_{2}\left(\partial \boldsymbol{\varphi}_{i j, e}, \boldsymbol{\varphi}_{k, e}\right) \partial \boldsymbol{\varphi}_{l m, e}+\overline{\mathbf{K}}_{2}\left(\boldsymbol{\varphi}_{i, e}, \partial \boldsymbol{\varphi}_{j k, e}\right) \partial \boldsymbol{\varphi}_{l m, e}+\right. \\
\left.\mathbf{K}_{2}\left(\partial \boldsymbol{\varphi}_{i j, e}, \partial \boldsymbol{\varphi}_{k l, e}\right) \boldsymbol{\varphi}_{m, e}\right) & \\
\mathbf{q}_{6, i j k l m n}^{*}= & \left(\boldsymbol{\Phi}_{T, e}\right)^{T} \overline{\mathbf{K}}_{2}\left(\partial \boldsymbol{\varphi}_{i j, e}, \partial \boldsymbol{\varphi}_{k l, e}\right) \partial \boldsymbol{\varphi}_{m n, e}
\end{aligned}
$$

where subindex $e$ on $\varphi_{i, e}$ and $\partial \varphi_{i j, e}$ refers to the local element considered. After evaluating the equivalent force vectors in (72)-(76) these are organized into the symmetry force vectors $\overline{\mathbf{q}}$. For (72) and (73) the relations in (64)-(65) can be reused. Fixed relations between $\mathbf{q}^{*}$ in (74)-(76) and $\overline{\mathbf{q}}$ also exist, but they are very comprehensive. The present authors, therefore, use a simple algorithm to organize the vectors $\overline{\mathbf{q}}$. The algorithm consists of a loop that runs over all contributions of $\mathbf{q}^{*}$ and checks if the values of the indexes $i$ to $n$ match previous vectors with those index values in any given combination. If this is the case, the vectors are added together.

The approach described above only determines the element local equivalent force vector coefficients. To obtain the global equivalent force vector coefficients, all element contributions are assembled.

\section{APPROXIMATE VELOCITY}

An approximation for the velocity vector $\dot{\mathbf{s}}^{(q)}$ at the discrete time $t^{(q)}=q \Delta t$ is arranged. For this, a second-order Taylor series of the displacement vector $\mathbf{s}$ is considered, with time $t^{(q)}$ taken as the point of reference

$$
\mathbf{s}(t)=\mathbf{s}^{(q)}+\dot{\mathbf{s}}^{(q)}\left(t-t^{(q)}\right)+\frac{1}{2} \ddot{\mathbf{s}}^{(q)}\left(t-t^{(q)}\right)^{2}
$$

The vectors $\dot{\mathbf{s}}^{(q)}$ and $\ddot{\mathbf{s}}^{(q)}$ are unknowns and should be determined. Both the displacement $\mathbf{s}^{(q-1)}$ and the velocity $\dot{\mathbf{s}}^{(q-1)}$ in the time state $t^{(q-1)}$ are assumed to be known. Two equations including the 
unknowns $\dot{\mathbf{s}}^{(q)}$ and $\ddot{\mathbf{s}}^{(q)}$ can be arranged by use of (77) evaluated at the time state $t^{(q-1)}$ as

$$
\begin{aligned}
& \mathbf{s}\left(t^{(q-1)}\right)=\mathbf{s}^{(q)}+\dot{\mathbf{s}}^{(q)}(-\Delta t)+\frac{1}{2} \ddot{\mathbf{s}}^{(q)}(-\Delta t)^{2}=\mathbf{s}^{(q-1)} \\
& \dot{\mathbf{s}}\left(t^{(q-1)}\right)=\dot{\mathbf{s}}^{(q)}+\ddot{\mathbf{s}}^{(q)}(-\Delta t)=\dot{\mathbf{s}}^{(q-1)}
\end{aligned}
$$

Then $\ddot{\mathbf{s}}^{(q)}$ is isolated in (79) and inserted it into (78). By this the desired velocity $\dot{\mathbf{s}}^{(q)}$ at time $t^{(q)}$ is expressed as a function of the previously evaluated displacements and velocities as:

$$
\dot{\mathbf{s}}^{(q)}=\frac{2}{\Delta t}\left(\mathbf{s}^{(q)}-\mathbf{s}^{(q-1)}\right)-\dot{\mathbf{s}}^{(q-1)}
$$

However, it is desired to evaluate the velocity solely on the basis of displacements. If the CDM is used, then the displacement vectors are explicitly related to the velocity by the expression

$$
\dot{\mathbf{s}}^{(q-1)}=\frac{1}{2 \Delta t}\left(\mathbf{s}^{(q)}-\mathbf{s}^{(q-2)}\right)
$$

Inserting (81) into (80) gives an approximation for the velocity vector at the time state $t^{(q)}$ in the form

$$
\dot{\mathbf{s}}^{(q)}=\frac{1}{\Delta t}\left(\frac{3}{2} \mathbf{s}^{(q)}-2 \mathbf{s}^{(q-1)}+\frac{1}{2} \mathbf{s}^{(q-2)}\right)
$$

The velocity approxmiation in (82) is purely based on the displacements at times $t^{(q)}, t^{(q-1)}$ and $t^{(q-2)}$.

\section{REFERENCES}

1. Barbic J, James D. Real-time subspace integration for st. venant-kirchhoff deformable models. ACM Transactions on Graphics 2005; 24:982-990, doi:10.1145/1073204.1073300.

2. Niroomandi S, Alfaro I, Cueto EG, Chinesta F. Accounting for large deformations in real-time simulations of soft tissues based on reduced-order models. Computer Methods and Programs In Boimedicine 2012; 105:1-12, doi: 10.1016/j.cmpb.2010.06.012.

3. Nakashima M, Masaoka N. Real-time on-line test for mdof systems. Earthquake Engineering \& Structural Dynamics 1999; 28:393-420, doi:10.1002/(sici)1096-9845(199904)28:4〈393::aid-eqe823〉3.0.co;2-c.

4. Hakuno M, Shidawara M, Hara T. Dynamic destructive test of a cantilever beam, controlled by an analog-computer (in japanese). Transactions of the Japan Society of Civil Engineers 1969; 1969:1-9, doi:10.2208/jscej1969.1969. 171_1.

5. Chen C, Ricles JM, Karavasilis TL, Chae Y, Sause R. Evaluation of a real-time hybrid simulation system for performance evaluation of structures with rate dependent devices subjected to seismic loading. Engineering Structures 2012; 35:71-82, doi:10.1016/j.engstruct.2011.10.006.

6. Verma M, Rajasankar J. Improved model for real-time substructuring testing system. Engineering Structures 2012; 41:258-269, doi:10.1016/j.engstruct.2012.03.031.

7. Yang YS, Tsai KC, Elnashai AS, Hsieh TJ. An online optimization method for bridge dynamic hybrid simulations. Simulation Modelling Practice and Theory 2012; 28:42-54, doi:10.1016/j.simpat.2012.06.002.

8. Abdelnaby AE, Frankie TM, Elnashai AS, Spencer BF, Kuchma DA, Silva P, Chang CM. Numerical and hybrid analysis of a curved bridge and methods of numerical model calibration. Engineering Structures 2014; 70:234-245, doi:10.1016/j.engstruct.2014.04.009.

9. Hashemi MJ, Masroor A, Mosqueda G. Implementation of online model updating in hybrid simulation. Earthquake Engineering and Structural Dynamics 2014; 43:395-412, doi:10.1002/eqe.2350.

10. Abbiati G, Bursi OS, Caperan P, Di Sarno L, Molina FJ, Paolacci F, Pegon P. Hybrid simulation of a multi-span rc viaduct with plain bars and sliding bearings. Earthquake Engineering and Structural Dynamics 2015; 44:22212240, doi:10.1002/eqe.2580.

11. Chae Y, Kazemibidokhti K, Ricles JM. Adaptive time series compensator for delay compensation of servo-hydraulic actuator systems for real-time hybrid simulation. Earthquake Engineering \& Structural Dynamics 2013; 42:16971715, doi:10.1002/eqe.2294.

12. Horri K, Kawahara M. A numerical analysis on the dynamic response of structures. Proceedings of 19th Japan National Congress for Applied Mechanics 1969; :17-22.

13. Darby AP, Blakeborough A, Williams MS. Improved control algorithm for real-time substructure testing. Earthquake Engineering \& Structural Dynamics 2001; 30:431-448, doi:10.1002/eqe.18.

14. Blakeborough A, Williams MS, Darby AP, Williams DM. The development of real-time substructure testing. Philosophical Transactions of the Royal Society A: Mathematical, Physical and Engineering Sciences 2001; 359:1869-1891, doi:10.1098/rsta.2001.0877. 
15. Lülf FA, Tran DM, Ohayon R. Reduced bases for nonlinear structural dynamic systems: A comparative study. Journal of Sound and Vibration 2013; 332:3897-3921, doi:10.1016/j.jsv.2013.02.014.

16. Idelsohn SR, Cardona A. A reduction method for nonlinear structural dynamic analysis. Computer Methods in Applied Mechanics and Engineering 1985; 49:253-279, doi:10.1016/0045-7825(85)90125-2.

17. Idelsohn SR, Cardona A. Recent advances in reduction methods in nonlinear structural dynamics. Proceedings of the Second International Conference on: Recent Advances in Structural Dynamics, vol. 2, University of Southampton, England, 1984; 475-482.

18. Idelsohn SR, Cardona A. A load-dependent basis for reduced nonlinear structural dynamics. Computers \& Structures 1985; 20:203-210, doi:10.1016/0045-7949(85)90069-0.

19. Weeger O, Wever U, Simeon B. Nonlinear frequency response analysis of structural vibrations. Computational Mechanics 2014; 54:1477-1495, doi:10.1007/s00466-014-1070-9.

20. Weeger O, Wever U, Simeon B. On the use of modal derivatives for nonlinear model order reduction. International Journals for Numerical Methods in Engineering 2016; 108:1579-1602, doi:10.1002/nme.5267.

21. Slaats PMA, de Jongh J, Sauren AAHJ. Model reduction tools for nonlinear structural dynamics. Computer \& Structures 1995 ; 54:1155-1171, doi:10.1016/0045-7949(94)00389-K.

22. Tiso P, Jansen E, Abdalla M. Reduction method for finite element nonlinear dynamic analysis of shells. Aiaa Journal 2011; 49:2295-2304, doi:10.2514/1.J051003.

23. Brake M, Segalman D. Nonlinear model reduction of von kármán plates under quasi-steady fluid flow. The American Institute of Aeronautics and Astronautics 2010; 48:2339-2347, doi:10.2514/1.J050357.

24. Nash M. Nonlinear structural dynamics by finite element modal synthesis. PhD Thesis, Imperial College, The University of London, Imperial College London 1978.

25. Shi Y, Mei C. A finite element time domain modal formulation for large amplitude free vibrations of beams and plates. Journal of Sound and Vibration 1996; 193:453-464, doi:10.1006/jsvi.1996.0295.

26. Spottswood S, Allemang R. Identification of nonlinear parameters for reduced order models. Journal of Sound and Vibration 2006; 295:226-245, doi:10.1016/j.jsv.2006.01.009.

27. Muravyov AA, Rizzi SA. Determination of nonlinear stiffness with application to random vibration of geometrically nonlinear structures. Computers \& Structures 2003; 81:1513-1523, doi:10.1016/S0045-7949(03) 00145-7.

28. Belytschko T, Liu WK, Moran B. Nonlinear Finite Elements for Continua and Structures. first edn., John Wiley \& Sons: Chicester, England, 2000.

29. Hahn G. A modified euler method for dynamic analysis. International Journal for Numerical Methods in Engineering 1991; 32:943-955, doi:10.1002/nme.1620320502.

30. Cook RD, Malkus DS, Plesha ME, Witt RJ. Concepts and Applications Of Finite Element Analysis. fourth edn., John Wiley \& Sons: New York, USA, 1974.

31. Chopra AK. Dynamics of Structures: Theory and Application to Earthquake Engineering. second edn., PrenticeHall: New Jersey, USA, 1995.

32. Poulsen PN, Damkilde L. A flat triangular shell element with loof nodes. International Journal for Numerical Methods in Engineering 1996; 39:3867-3887, doi:10.1002/(SICI)1097-0207(19961130)39:22<3867:: AID-NME28>3.0.CO;2-H. 